\title{
Generalized fractional maximal and integral operators on Orlicz and generalized Orlicz-Morrey spaces of the third kind
}

\author{
Fatih Deringoz, Vagif S. Guliyev, Eiichi Nakai, \\ Yoshihiro Sawano and Minglei Shi
}

\begin{abstract}
In the present paper, we will characterize the boundedness of the generalized fractional integral operators $I_{\rho}$ and the generalized fractional maximal operators $M_{\rho}$ on Orlicz spaces, respectively. Moreover, we will give a characterization for the Spanne-type boundedness and the Adams-type boundedness of the operators $M_{\rho}$ and $I_{\rho}$ on generalized Orlicz-Morrey spaces, respectively. Also we give criteria for the weak versions of the Spanne-type boundedness and the Adams-type boundedness of the operators $M_{\rho}$ and $I_{\rho}$ on generalized Orlicz-Morrey spaces.
\end{abstract}

\section{Introduction}

The aim of this paper is to obtain the necessary conditions and the sufficient condtions for the generalized fractional maximal operator $M_{\rho}$ and the generalized fractional integral operator $I_{\rho}$ to be bounded on Orlicz spaces. Our results can be extended to generalized Orlicz-Morrey spaces of the third kind which will be defined later in this paper.

Let $\mathbb{R}^{n}$ be the $n$-dimensional Euclidean space. For a function $\rho:(0, \infty) \rightarrow$ $(0, \infty)$, let $I_{\rho}$ be the generalized fractional integral operator:

$$
I_{\rho} f(x)=\int_{\mathbb{R}^{n}} \frac{\rho(|x-y|)}{|x-y|^{n}} f(y) d y
$$

Here $f$ is a suitable measurable function. Note that this type of generalization goes back to [25]. If $\rho(r)=r^{\alpha}, 0<\alpha<n$, then $I_{\rho}$ is the fractional integral operator or

2010 Mathematics Subject Classification. 42B20, 42B25, 42B35, 46E30

Key words and phrases. generalized fractional maximal function, generalized fractional integral, Orlicz spaces, generalized Orlicz-Morrey spaces 
the Riesz potential and denoted by $I_{\alpha}$. Hereafter, we assume that

$$
\int_{0}^{1} \frac{\rho(t)}{t} d t<\infty
$$

so that the fractional integrals $I_{\rho} f$ are well defined, at least for characteristic functions of balls. The operator $I_{\rho}$ was introduced in [19] and some partial results were announced in [18]. We refer to [16] for the boundedness of $I_{\rho}$ on Orlicz space $L^{\Phi}(\Omega)$ with bounded domain $\Omega \subset \mathbb{R}^{n}$. See also [20, 21, 22, 23] for the boundedness of $I_{\rho}$ on various spaces. In these papers we assumed that $\rho$ satisfies the doubling condition:

$$
\frac{1}{C_{1}} \leq \frac{\rho(r)}{\rho(s)} \leq C_{1}, \quad \text { if } \frac{1}{2} \leq \frac{r}{s} \leq 2
$$

and that $r \mapsto \rho(r) / r^{n}$ is almost decreasing:

$$
\frac{\rho(s)}{s^{n}} \leq C_{2} \frac{\rho(r)}{r^{n}}, \quad \text { if } r<s,
$$

where $C_{1}$ and $C_{2}$ are positive constants independent of $r, s \in(0, \infty)$. Under these conditions we proved the boundedness of $I_{\rho}$ on Orlicz spaces in [18, 19].

In this paper, instead of these conditions, we assume that there exist positive constants $C, k_{1}$ and $k_{2}$ with $k_{1}<k_{2}$ such that, for all $r>0$,

$$
\sup _{r / 2 \leq t \leq r} \rho(t) \leq C \int_{k_{1} r}^{k_{2} r} \frac{\rho(t)}{t} d t=: \tilde{\rho}(r) .
$$

The condition (1.4) was considered in [26] and also used in [31]. If $\rho$ satisfies (1.2) or (1.3), then $\rho$ satisfies (1.4). Let

$$
\rho(r)= \begin{cases}r^{n}(\log (e / r))^{-1 / 2}, & 0<r<1, \\ e^{-(r-1)}, & 1 \leq r<\infty .\end{cases}
$$

Then $\rho$ satisfies (1.1) and (1.4), but fails (1.2) and (1.3). Therefore, the results in this paper improve ones in [19]. Moreover, we give necessary and sufficient conditions for the boundedness of $I_{\rho}$ not only on Orlicz spaces but also on OrliczMorrey spaces of the third kind.

Next, we define the generalized fractional maximal operator $M_{\rho}$. For a function $\rho:(0, \infty) \rightarrow(0, \infty)$, let

$$
M_{\rho} f(x)=\sup _{r>0} \frac{\rho(r)}{|B(x, r)|} \int_{B(x, r)}|f(y)| d y,
$$

where $|G|$ is the Lebesgue measure of a measurable set $G \subset \mathbb{R}^{n}$. We do not assume (1.1) on the function $\rho$ in (1.6). Instead we suppose that $\rho$ is an increasing function such that $r \in(0, \infty) \mapsto r^{-n} \rho(r) \in(0, \infty)$ is decreasing. 
If $\rho \equiv 1$, then $M_{\rho}$ is the Hardy-Littlewood maximal operator denoted by $M$. If $\rho(r)=r^{\alpha}$, then $M_{\rho}$ is the usual fractional maximal operator denoted by $M_{\alpha}$. We give some necessary conditions and some sufficient conditions for the boundedness of $M_{\rho}$ on Orlicz and Orlicz-Morrey spaces.

The structure of the remaining part of the present paper is as follows: First we recall Young functions and Orlicz spaces in Section 2, In Section 3, we investigate the boundedness of generalized fractional integrals on Orlicz spaces. We will give a necessary and sufficient condition for the boundedness of the generalized fractional maximal operators on Orlicz spaces in Section 4. In Section 5 we discuss some properties of generalized Orlicz-Morrey spaces of the third kind. Moreover, we will give necessary and sufficient conditions for the Spanne and Adams-type boundedness of the generalized fractional integral operators on generalized Orlicz-Morrey spaces of the third kind in Section 6. Finally, in Section 7 we give criteria for the boundedness of the generalized fractional maximal operators on generalized Orlicz-Morrey spaces of the third kind.

\section{Young functions and Orlicz spaces}

We recall the definition of Young functions.

Definition 2.1. A function $\Phi:[0, \infty] \rightarrow[0, \infty]$ is called a Young function if $\Phi$ is convex, left-continuous, $\lim _{r \rightarrow+0} \Phi(r)=\Phi(0)=0$ and $\lim _{r \rightarrow \infty} \Phi(r)=\Phi(\infty)=\infty$.

From the non-negativity, convexity and $\Phi(0)=0$ it follows that any Young function is increasing. We denote by $\mathcal{Y}$ the set of all Young functions such that

$$
0<\Phi(r)<\infty \quad \text { for } \quad 0<r<\infty .
$$

If $\Phi \in \mathcal{Y}$, then $\Phi$ is absolutely continuous on every compact interval in $[0, \infty)$ and bijective from $[0, \infty)$ to itself.

Next we recall the generalized inverse of Young function $\Phi$ in the sense of O'Neil [24, Definition 1.2]. For a Young function $\Phi$ and $0 \leq s \leq \infty$, let

$$
\Phi^{-1}(s)=\inf \{r \geq 0: \Phi(r)>s\}, \quad \text { where } \inf \emptyset=\infty .
$$

Note that if $s<\infty$, then so is $\Phi^{-1}(s)$. As in [24, p. 301, Remarks], we always have $\Phi^{-1}(\infty)=\infty$. An important inequality we use is

$$
\Phi\left(\Phi^{-1}(r)\right) \leq r \leq \Phi^{-1}(\Phi(r)) .
$$

See [24, Property 1.3]. Then $\Phi^{-1}(s)$ is finite for all $s \in[0, \infty)$, continuous on $(0, \infty)$ and right continuous at $s=0$. Observe that $\Phi^{-1}(\Phi(r))=r$ if $0<\Phi(r)<\infty$ and that $\Phi\left(\Phi^{-1}(s)\right)=s$ if $s=\Phi(r) \in(0, \infty)$ if $s \in[0, \Phi(\inf \{r>0: \Phi(r)=\infty\})]$. Furthermore, if $\Phi \in \mathcal{Y}$, then $\Phi^{-1}$ is the usual inverse function of $\Phi$. 
Remark 2.2. For a Young function $\Phi$, its inverse function $\Phi^{-1}$ is increasing and concave. Hence, we have the following properties:

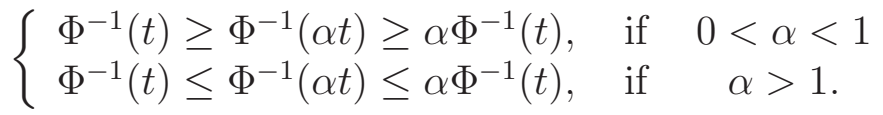

Since $\Phi^{-1}$ is increasing, the left inequality is clear. In particular, $\Phi$ satisfies the doubling condition: $\Phi^{-1}(2 s) \leq 2 \Phi^{-1}(s)$ for all $s \geq 0$.

In fact for $0<\alpha<1$,

$$
\Phi^{-1}(\alpha t)=\inf \{s \geq 0: \Phi(s)>\alpha t\} .
$$

Since $\frac{1}{\alpha} \Phi(s) \leq \Phi\left(\frac{s}{\alpha}\right)$, we have

$$
\Phi^{-1}(\alpha t) \geq \inf \left\{s \geq 0: \Phi\left(\frac{s}{\alpha}\right)>\alpha t\right\}=\alpha \inf \{s \geq 0: \Phi(s)>t\}=\alpha \Phi^{-1}(t) .
$$

The right inequality for $\alpha>1$ is a consequence of the one for $0<\alpha<1$.

As in [24, Property 1.6], we have

$$
r \leq \Phi^{-1}(r) \widetilde{\Phi}^{-1}(r) \leq 2 r \quad \text { for } r \geq 0,
$$

where $\widetilde{\Phi}(r)$ is the complementary function of $\Phi$ defined by

$$
\widetilde{\Phi}(r)=\left\{\begin{array}{cc}
\sup \{r s-\Phi(s): s \in[0, \infty)\} & , \quad r \in[0, \infty) \\
\infty & , \quad r=\infty
\end{array}\right.
$$

Then $\widetilde{\Phi}$ is also a Young function and $\widetilde{\widetilde{\Phi}}=\Phi$.

A Young function $\Phi$ is said to satisfy the $\Delta_{2}$-condition, denoted also by $\Phi \in \Delta_{2}$, if

$$
\Phi(2 r) \leq C \Phi(r), \quad r>0
$$

for some $C \geq 1$. If $\Phi \in \Delta_{2}$, then $\Phi \in \mathcal{Y}$. A Young function $\Phi$ is said to satisfy the $\nabla_{2}$-condition, denoted also by $\Phi \in \nabla_{2}$, if

$$
\Phi(r) \leq \frac{1}{2 C} \Phi(C r), \quad r \geq 0
$$

for some $C>1$.

We denote by $\chi_{G}$ the characteristic function of the set $G \subset \mathbb{R}^{n}$.

Definition 2.3 (Orlicz Space). For a Young function $\Phi$, the Orlicz space $L^{\Phi}\left(\mathbb{R}^{n}\right)$ is defined by:

$$
L^{\Phi}\left(\mathbb{R}^{n}\right)=\left\{f \in L_{\mathrm{loc}}^{1}\left(\mathbb{R}^{n}\right): \int_{\mathbb{R}^{n}} \Phi(k|f(x)|) d x<\infty \text { for some } k>0\right\} .
$$

The space $L_{\text {loc }}^{\Phi}\left(\mathbb{R}^{n}\right)$ is defined as the set of all measurable functions $f$ such that $f \chi_{B} \in L^{\Phi}\left(\mathbb{R}^{n}\right)$ for all balls $B \subset \mathbb{R}^{n}$. 
If $\Phi$ is a Young function, then $L^{\Phi}\left(\mathbb{R}^{n}\right)$ is a Banach space under the LuxemburgNakano norm

$$
\|f\|_{L^{\Phi}}=\inf \left\{\lambda>0: \int_{\mathbb{R}^{n}} \Phi\left(\frac{|f(x)|}{\lambda}\right) d x \leq 1\right\} .
$$

For example, if $\Phi(r)=r^{p}, 1 \leq p<\infty$, then $L^{\Phi}\left(\mathbb{R}^{n}\right)=L^{p}\left(\mathbb{R}^{n}\right)$. If $\Phi(r)=0,(0 \leq$ $r \leq 1)$ and $\Phi(r)=\infty,(r>1)$, then $L^{\Phi}\left(\mathbb{R}^{n}\right)=L^{\infty}\left(\mathbb{R}^{n}\right)$.

For a measurable set $\Omega \subset \mathbb{R}^{n}$, a measurable function $f$ and $t>0$, let

$$
m(\Omega, f, t)=|\{x \in \Omega:|f(x)|>t\}| .
$$

In the case $\Omega=\mathbb{R}^{n}$, we abbreviate it to $m(f, t)$.

Let $L^{0}\left(\mathbb{R}^{n}\right)$ be the set of all measurable functions.

Definition 2.4. For a Young function $\Phi$, the weak Orlicz space

$$
\mathrm{W} L^{\Phi}\left(\mathbb{R}^{n}\right)=\left\{f \in L^{0}\left(\mathbb{R}^{n}\right):\|f\|_{\mathrm{W} L^{\Phi}}<\infty\right\}
$$

is defined by the quasi-norm

$$
\|f\|_{\mathrm{W} L^{\Phi}}=\sup _{\lambda>0}\left\|\lambda \chi_{(\lambda, \infty)}(|f|)\right\|_{L^{\Phi}} .
$$

For $\Omega \subset \mathbb{R}^{n}$, let

$$
\|f\|_{L^{\Phi}(\Omega)}:=\left\|f \chi_{\Omega}\right\|_{L^{\Phi}} \text { and } \quad\|f\|_{\mathrm{W} L^{\Phi}(\Omega)}:=\left\|f \chi_{\Omega}\right\|_{\mathrm{W} L^{\Phi}} .
$$

A tacit understanding is that $f$ is defined to be zero outside $\Omega$.

We note that $\|f\|_{\mathrm{W} L^{\Phi}(\Omega)} \leq\|f\|_{L^{\Phi}(\Omega)}$, that

$$
\begin{aligned}
\|f\|_{\mathrm{W} L^{\Phi}} & =\sup _{t>0} \Phi(t) m(\Omega, f, t) \\
& =\sup _{t>0} t m\left(\Omega, f, \Phi^{-1}(t)\right) \\
& =\sup _{t>0} t m(\Omega, \Phi(|f|), t)
\end{aligned}
$$

and that

$$
\int_{\Omega} \Phi\left(\frac{|f(x)|}{\|f\|_{L^{\Phi}(\Omega)}}\right) d x \leq 1, \quad \sup _{t>0} \Phi(t) m\left(\Omega, \frac{f}{\|f\|_{\mathrm{W} L^{\Phi}(\Omega)}}, t\right) \leq 1
$$

according to [13, Proposition 4.2].

The following analogue of the Hölder inequality is well known; see [32] as well as the paper [24, §II] and the textbooks [14, 29].

Theorem 2.5. Let $\Omega \subset \mathbb{R}^{n}$ be a measurable set and, let $f$ and $g$ be measurable functions on $\Omega$. For a Young function $\Phi$ and its complementary function $\widetilde{\Phi}$, the following inequality is valid:

$$
\int_{\Omega}|f(x) g(x)| d x \leq 2\|f\|_{L^{\Phi}(\Omega)}\|g\|_{L^{\widetilde{\Phi}(\Omega)}} .
$$


By elementary calculations we have the following property:

Lemma 2.6. Let $\Phi$ be a Young function and let $B$ be a set in $\mathbb{R}^{n}$ with finite Lebesgue measure. Then

$$
\left\|\chi_{B}\right\|_{L^{\Phi}}=\left\|\chi_{B}\right\|_{\mathrm{W} L^{\Phi}}=\frac{1}{\Phi^{-1}\left(|B|^{-1}\right)} .
$$

By Theorem 2.5, Lemma 2.6 and (2.1) we get the following estimate:

Lemma 2.7. For a Young function $\Phi$ and $B=B(x, r)$, the following inequality is valid:

$$
\int_{B}|f(y)| d y \leq 2|B| \Phi^{-1}\left(|B|^{-1}\right)\|f\|_{L^{\Phi}(B)}
$$

We recall the boundedness property of the Hardy-Littlewood maximal operator $M$ on Orlicz spaces since we use it later.

Theorem 2.8. Let $\Phi$ be a Young function.

1. [2, Theorem 1] The operator $M$ is bounded from $L^{\Phi}\left(\mathbb{R}^{n}\right)$ to $\mathrm{W} L^{\Phi}\left(\mathbb{R}^{n}\right)$, and the inequality

$$
\|M f\|_{\mathrm{W} L^{\Phi}} \leq C_{0}\|f\|_{L^{\Phi}}
$$

holds with constant $C_{0}$ independent of $f$.

2. [2, Theorem 1], [11, Corollary 3.3] The operator $M$ is bounded on $L^{\Phi}\left(\mathbb{R}^{n}\right)$, and the inequality

$$
\|M f\|_{L^{\Phi}} \leq C_{0}\|f\|_{L^{\Phi}}
$$

holds with constant $C_{0}$ independent of $f$ if and only if $\Phi \in \nabla_{2}$.

See the textbooks [14, 15, 27, 29] for more about Orlicz spaces.

\section{$3 \quad$ Generalized fractional integrals on Orlicz spaces}

The following theorem is one of our main results and gives necessary and sufficient conditions for the boundedness of the operator $I_{\rho}$ from $L^{\Phi}\left(\mathbb{R}^{n}\right)$ to $\mathrm{W} L^{\Psi}\left(\mathbb{R}^{n}\right)$ and from $L^{\Phi}\left(\mathbb{R}^{n}\right)$ to $L^{\Psi}\left(\mathbb{R}^{n}\right)$.

Theorem 3.1. Let $\Phi, \Psi$ be Young functions.

1. Let $\rho$ satisfy the conditions (1.1) and (1.4). Then the condition

$$
\Phi^{-1}\left(r^{-n}\right) \int_{0}^{r} \frac{\rho(t)}{t} d t+\int_{r}^{\infty} \rho(t) \Phi^{-1}\left(t^{-n}\right) \frac{d t}{t} \leq C \Psi^{-1}\left(r^{-n}\right)
$$

for all $r>0$, where $C>0$ does not depend on $r$, is sufficient for the boundedness of $I_{\rho}$ from $L^{\Phi}\left(\mathbb{R}^{n}\right)$ to $\mathrm{W} L^{\Psi}\left(\mathbb{R}^{n}\right)$. Moreover, if $\Phi \in \nabla_{2}$, then the condition (3.1) is also sufficient for the boundedness of $I_{\rho}$ from $L^{\Phi}\left(\mathbb{R}^{n}\right)$ to $L^{\Psi}\left(\mathbb{R}^{n}\right)$. 
2. The condition

$$
\Phi^{-1}\left(r^{-n}\right) \int_{0}^{r} \frac{\rho(t)}{t} d t \leq C \Psi^{-1}\left(r^{-n}\right)
$$

for all $r>0$, where $C>0$ does not depend on $r$, is necessary for the boundedness of $I_{\rho}$ from $L^{\Phi}\left(\mathbb{R}^{n}\right)$ to $\mathrm{W} L^{\Psi}\left(\mathbb{R}^{n}\right)$ and from $L^{\Phi}\left(\mathbb{R}^{n}\right)$ to $L^{\Psi}\left(\mathbb{R}^{n}\right)$.

3. Let $\rho$ satisfy the conditions (1.1) and (1.4). Assume the condition

$$
\int_{r}^{\infty} \rho(t) \Phi^{-1}\left(t^{-n}\right) \frac{d t}{t} \leq C \Psi^{-1}\left(r^{-n}\right)
$$

holds for all $r>0$, where $C>0$ does not depend on $r$. Then condition (3.2) is necessary and sufficient for the boundedness of $I_{\rho}$ from $L^{\Phi}\left(\mathbb{R}^{n}\right)$ to $\mathrm{W} L^{\Psi}\left(\mathbb{R}^{n}\right)$. Moreover, if $\Phi \in \nabla_{2}$, then the condition (3.2) is necessary and sufficient for the boundedness of $I_{\rho}$ from $L^{\Phi}\left(\mathbb{R}^{n}\right)$ to $L^{\Psi}\left(\mathbb{R}^{n}\right)$.

Remark 3.2. We cannot replace $\int_{0}^{r} \frac{\rho(t)}{t} d t$ by $\rho(r)$ in (3.1), see [23, Section 5].

We need a couple of auxilary estimates. The following lemma was proved in 8 , Lemma 2.1]:

Lemma 3.3. There exist a constant $C>0$ such that for all $x \in B(0, r / 2)$ and $r>0$,

$$
\int_{0}^{r / 2} \frac{\rho(t)}{t} d t \leq C I_{\rho} \chi_{B(0, r)}(x)
$$

holds.

Proposition 3.4. Let $\rho$ satisfy (1.4). Define

$$
\tilde{\rho}(r)=\int_{k_{1} r}^{k_{2} r} \rho(s) \frac{d s}{s} \quad(r>0) .
$$

Let $\tau:(0, \infty) \rightarrow(0, \infty)$ be a doubling function in the sense that $\tau(r) \sim \tau(s)$ if $0<s \leq r \leq 2 s$. Then, for each $r>0$,

$$
\begin{aligned}
\sum_{j=-\infty}^{-1} \tilde{\rho}\left(2^{j} r\right) & \lesssim \int_{0}^{k_{2} r} \frac{\rho(s)}{s} d s \\
\sum_{j=0}^{\infty} \tilde{\rho}\left(2^{j} r\right) \tau\left(\left(2^{j} r\right)^{-n}\right) & \lesssim \int_{k_{1} r}^{\infty} \frac{\rho(s)}{s} \tau\left(s^{-n}\right) d s
\end{aligned}
$$


Proof. We invoke the overlapping property in 31] and by Remark 2.2 we have

$$
\begin{aligned}
\sum_{j=-\infty}^{-1} \tilde{\rho}\left(2^{j} r\right) & =\sum_{j=-\infty}^{-1} \int_{2^{j} k_{1} r}^{2^{j} k_{2} r} \rho(s) \frac{d s}{s} \\
& \leq \int_{0}^{k_{2} r}\left(\sum_{j=-\infty}^{-1} \chi_{\left[2^{j} k_{1} r, 2^{j} k_{2} r\right]}(s)\right) \frac{\rho(s)}{s} d s \\
& \lesssim \int_{0}^{k_{2} r} \frac{\rho(s)}{s} d s
\end{aligned}
$$

and

$$
\begin{aligned}
\sum_{j=0}^{\infty} \tilde{\rho}\left(2^{j} r\right) \tau\left(\left(2^{j} r\right)^{-n}\right) & =\int_{k_{1} r}^{\infty}\left(\sum_{j=0}^{\infty} \chi_{\left[2^{j} k_{1} r, 2^{j} k_{2} r\right]}(s) \frac{\rho(s)}{s} \tau\left(\left(2^{j} r\right)^{-n}\right)\right) d s \\
& \lesssim \int_{k_{1} r}^{\infty}\left(\sum_{j=0}^{\infty} \chi_{\left[2^{j} k_{1} r, 2^{j} k_{2} r\right]}(s)\right) \frac{\rho(s)}{s} \tau\left(s^{-n}\right) d s \\
& \lesssim \int_{k_{1} r}^{\infty} \frac{\rho(s)}{s} \tau\left(s^{-n}\right) d s .
\end{aligned}
$$

To prove Theorem 3.1, we need the following estimate of Hedberg-type [12]:

Proposition 3.5. Under the assumption of Theorem 3.1, for any positive constant $C_{0}$, there exists a positive constant $C_{1}$ such that, for all nonnegative functions $f \in$ $L^{\Phi}\left(\mathbb{R}^{n}\right)$ with $f \neq 0$,

$$
I_{\rho} f(x) \leq C_{1}\|f\|_{L^{\Phi}} \Psi^{-1} \circ \Phi\left(\frac{M f(x)}{C_{0}\|f\|_{L^{\Phi}}}\right) \quad\left(x \in \mathbb{R}^{n}\right) .
$$

Proof. The idea of the proof comes from [8]. First note that

$$
0<\Phi^{-1}(0) \int_{0}^{\infty} \frac{\rho(t)}{t} d t \lesssim \Psi^{-1}(0)
$$

as long as $\Phi^{-1}(0)>0$.

Let $x \in \mathbb{R}^{n}$. Keeping in mind that $M f(x)>0$, we may assume

$$
0<\frac{M f(x)}{C_{0}\|f\|_{L^{\Phi}}}<\infty, \quad 0 \leq \Phi\left(\frac{M f(x)}{C_{0}\|f\|_{L^{\Phi}}}\right)<\infty
$$

otherwise there is nothing to prove. If

$$
\Phi\left(\frac{M f(x)}{C_{0}\|f\|_{L^{\Phi}}}\right)=0
$$


then

$$
\frac{M f(x)}{C_{0}\|f\|_{L^{\Phi}}} \leq \sup \{u \geq 0: \Phi(u)=0\}=\Phi^{-1}(0)
$$

and hence

$$
\begin{aligned}
I_{\rho} f(x) & \leq C \sum_{j=-\infty}^{\infty} \frac{\tilde{\rho}\left(2^{j}\right)}{2^{j n}} \int_{|x-y|<2^{j}}|f(y)| d y \\
& \leq C\left(\int_{0}^{\infty} \frac{\rho(s)}{s} d s\right) M f(x) \\
& \leq C \frac{\Psi^{-1}(0)}{\Phi^{-1}(0)} M f(x) \\
& \leq C \frac{1}{\Phi^{-1}(0)} \Psi^{-1}\left(\Phi\left(\frac{M f(x)}{C_{0}\|f\|_{L^{\Phi}}}\right)\right) M f(x) \\
& \leq C \Psi^{-1}\left(\Phi\left(\frac{M f(x)}{C_{0}\|f\|_{L^{\Phi}}}\right)\right)\|f\|_{L^{\Phi}} .
\end{aligned}
$$

So, this case the result is valid.

If

$$
\Phi\left(\frac{M f(x)}{C_{0}\|f\|_{L^{\Phi}}}\right)>0
$$

choose $r \in(0, \infty)$ so that

$$
r^{-n}=\Phi\left(\frac{M f(x)}{C_{0}\|f\|_{L^{\Phi}}}\right)
$$

We have

$$
I_{\rho} f(x) \leq C\left[\sum_{j=-\infty}^{-1}+\sum_{j=0}^{\infty} \frac{\tilde{\rho}\left(2^{j} r\right)}{\left(2^{j} r\right)^{n}} \int_{|x-y|<2^{j} r} f(y) d y\right]=C(\mathrm{I}+\mathrm{II})
$$

for given $x \in \mathbb{R}^{n}$ and $r>0$.

Then from Proposition 3.4

$$
\begin{aligned}
\mathrm{I} & \leq C \sum_{j=-\infty}^{-1} \tilde{\rho}\left(2^{j} r\right) M f(x) \leq C\left(\int_{0}^{k_{2} r} \frac{\rho(s)}{s} d s\right) M f(x) \\
\mathrm{II} & \leq C \sum_{j=0}^{\infty} \tilde{\rho}\left(2^{j} r\right) \Phi^{-1}\left(\left(2^{j} r\right)^{-n}\right)\|f\|_{L^{\Phi}\left(B\left(x, 2^{j} r\right)\right)} \\
& \leq C\|f\|_{L^{\Phi}} \int_{k_{1} r}^{\infty} \Phi^{-1}\left(s^{-n}\right) \frac{\rho(s)}{s} d s .
\end{aligned}
$$

Consequently, we have

$$
I_{\rho} f(x) \lesssim\left(\int_{0}^{k_{2} r} \frac{\rho(s)}{s} d s\right) M f(x)+\|f\|_{L^{\Phi}} \int_{k_{1} r}^{\infty} \Phi^{-1}\left(s^{-n}\right) \frac{\rho(s)}{s} d s .
$$


Thus, by the doubling property of $\Phi^{-1}$ and $\Psi^{-1}$, (3.1) and Remark 2.2 we obtain

$$
\begin{aligned}
I_{\rho} f(x) & \lesssim M f(x) \frac{\Psi^{-1}\left(\left(k_{2} r\right)^{-n}\right)}{\Phi^{-1}\left(\left(k_{2} r\right)^{-n}\right)}+\|f\|_{L^{\Phi}} \Psi^{-1}\left(\left(k_{1} r\right)^{-n}\right) \\
& \lesssim M f(x) \frac{\Psi^{-1}\left(r^{-n}\right)}{\Phi^{-1}\left(r^{-n}\right)}+\|f\|_{L^{\Phi}} \Psi^{-1}\left(r^{-n}\right) .
\end{aligned}
$$

Recall that $\Phi^{-1}(\Phi(r))=r$ if $0<\Phi(r)<\infty$. Thus $\Phi^{-1}\left(r^{-n}\right)=\frac{M f(x)}{C_{0}\|f\|_{L^{\Phi}}}$ and

$$
I_{\rho} f(x) \lesssim\|f\|_{L^{\Phi}} \Psi^{-1}\left(r^{-n}\right)=\|f\|_{L^{\Phi}} \Psi^{-1}\left(\Phi\left(\frac{M f(x)}{C_{0}\|f\|_{L^{\Phi}}}\right)\right) .
$$

Therefore, we get (3.7).

Now we move on to the proof of Theorem 3.1. The third statement is a consequence of the remaining statements. So we concentrate on the first and the second ones.

- Let $C_{0}$ be as in (2.3). Let $f$ be a non-negative measurable function. Then by (2.3) and (3.7),

$$
\begin{aligned}
& \sup _{r>0} \Psi(r) m\left(\frac{I_{\rho} f(x)}{C_{1}\|f\|_{L^{\Phi}}}, r\right)=\sup _{r>0} r m\left(\Psi\left(\frac{I_{\rho} f(x)}{C_{1}\|f\|_{L^{\Phi}}}\right), r\right) \\
\leq & \sup _{r>0} r m\left(\Phi\left(\frac{M f(x)}{C_{0}\|f\|_{L^{\Phi}}}\right), r\right) \leq \sup _{r>0} \Phi(r) m\left(\frac{M f(x)}{\|M f\|_{\mathrm{W} L^{\Phi}}}, r\right) \leq 1,
\end{aligned}
$$

i.e.

$$
\left\|I_{\rho} f\right\|_{\mathrm{W} L^{\Psi}} \lesssim\|f\|_{L^{\Phi}}
$$

- Assume in addition that $\Phi \in \nabla_{2}$, so that we have (2.4). By (2.4), we have

$$
\begin{aligned}
\int_{\mathbb{R}^{n}} \Psi\left(\frac{I_{\rho} f(x)}{C_{1}\|f\|_{L^{\Phi}}}\right) d x & \leq \int_{\mathbb{R}^{n}} \Phi\left(\frac{M f(x)}{C_{0}\|f\|_{L^{\Phi}}}\right) d x \\
& \leq \int_{\mathbb{R}^{n}} \Phi\left(\frac{M f(x)}{\|M f\|_{L^{\Phi}}}\right) d x \leq 1
\end{aligned}
$$

i.e.

$$
\left\|I_{\rho} f\right\|_{L^{\Psi}} \lesssim\|f\|_{L^{\Phi}}
$$

- We can and do concentrate on the boundedness of $I_{\rho}$ from $L^{\Phi}\left(\mathbb{R}^{n}\right)$ to W $L^{\Psi}\left(\mathbb{R}^{n}\right)$, since the boundedness of $I_{\rho}$ from $L^{\Phi}\left(\mathbb{R}^{n}\right)$ to $L^{\Psi}\left(\mathbb{R}^{n}\right)$ is stronger than the boundedness of $I_{\rho}$ from $L^{\Phi}\left(\mathbb{R}^{n}\right)$ to $\mathrm{W} L^{\Psi}\left(\mathbb{R}^{n}\right)$. With this in mind, assume that $I_{\rho}$ is bounded from $L^{\Phi}\left(\mathbb{R}^{n}\right)$ to $W L^{\Psi}\left(\mathbb{R}^{n}\right)$. 
Then we have by Lemma 3.3

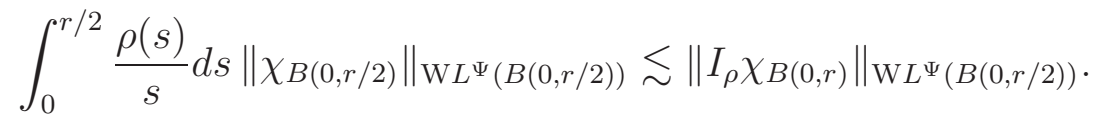

Therefore, by the doubling property of $\Phi^{-1}$ and Lemma 2.6, we have

$$
\begin{aligned}
\int_{0}^{r / 2} \frac{\rho(s)}{s} d s & \lesssim \Psi^{-1}\left(r^{-n}\right)\left\|I_{\rho} \chi_{B(0, r)}\right\|_{\mathrm{W} L^{\Psi}(B(0, r / 2))} \\
& \lesssim \Psi^{-1}\left(r^{-n}\right)\left\|I_{\rho} \chi_{B(0, r)}\right\|_{\mathrm{W} L^{\Psi}} \\
& \lesssim \Psi^{-1}\left(r^{-n}\right)\left\|\chi_{B(0, r)}\right\|_{L^{\Phi}} \\
& \lesssim \frac{\Psi^{-1}\left(r^{-n}\right)}{\Phi^{-1}\left(r^{-n}\right)}
\end{aligned}
$$

Remark 3.6. In [19, Corollary 3.2] the third author found the sufficient conditions which ensures the boundedness of the operator $I_{\rho}$ from $L^{\Phi}\left(\mathbb{R}^{n}\right)$ to $L^{\Psi}\left(\mathbb{R}^{n}\right)$, including its weak version. Theorem 3.1 improves the third author's result in that Theorem 3.1 also covers the necessity by imposing a weaker condition on $\rho$.

Remark 3.7. In the case $\Phi(t)=t^{p}$, Theorem [3.1 was proved in [8, Corollary 1.5].

Example 3.8. Let $\rho$ be as in (1.5) and

$$
\Phi(t)=\left\{\begin{array}{ll}
t^{3 / 2}, & 0 \leq t \leq 1, \\
t(\log (e t))^{1 / 2}, & t>1,
\end{array} \quad \Psi(t)= \begin{cases}\frac{2 e}{3} t^{3 / 2}, & 0 \leq t \leq 1 \\
\frac{2 e}{3} \frac{\exp \exp (t)}{\exp \exp (1)}, & t>1\end{cases}\right.
$$

Then the pair $(\rho, \Phi, \Psi)$ satisfies (3.1). In fact, we have

$$
\begin{aligned}
\Phi^{-1}(u) & \sim \begin{cases}u^{2 / 3}, & 0 \leq u \leq 1, \\
u(\log (e u))^{-1 / 2}, & u>1,\end{cases} \\
\Psi^{-1}(u) & \sim \begin{cases}u^{2 / 3}, & 0 \leq u \leq 1, \\
\log \left(\log \left(e^{e} u\right)\right), & u>1,\end{cases}
\end{aligned}
$$

and, for all $r>0$,

$$
\begin{gathered}
\int_{0}^{r} \frac{\rho(t)}{t} d t \Phi^{-1}\left(1 / r^{n}\right) \lesssim \min \left(1, r^{-2 n / 3}\right), \\
\int_{r}^{\infty} \frac{\rho(t) \Phi^{-1}\left(1 / t^{n}\right)}{t} d t \lesssim \min \left(\log \log \left(e^{e} / r\right), r^{-2 n / 3}\right) \\
\Psi^{-1}\left(1 / r^{n}\right) \sim \min \left(\log \log \left(e^{e} / r\right), r^{-2 n / 3}\right)
\end{gathered}
$$

See [20] for other examples. 


\section{Generalized fractional maximal operators on Orlicz spaces}

We recall that, for a function $\rho:(0, \infty) \rightarrow(0, \infty), M_{\rho}$ is defined by (1.6). Here we suppose that $\rho$ is an increasing function such that $r \in(0, \infty) \mapsto r^{-n} \rho(r) \in(0, \infty)$ is decreasing.

Under this assumption, we have the following localized estimate:

Lemma 4.1. There exists a positive constant $C$ such that, for all balls $B=B(x, r)$ and all measurable functions $f$ supported on $B$,

$$
M_{\rho} f(x) \leq C \rho(r) M f(x) .
$$

Proof. Let $B(R)=B(x, R)$ with $x=0$ for $R>0$. By the definition of $M_{\rho}$, we have

$$
\begin{aligned}
& M_{\rho} f(x) \\
& =\max \left\{\sup _{0<R<3 r} \frac{\rho(R)}{|B(R)|} \int_{B(x, R)}|f(y)| d y, \sup _{R \geq 3 r} \frac{\rho(R)}{|B(R)|} \int_{B(x, R)}|f(y)| d y\right\} .
\end{aligned}
$$

For the first term, we use the fact that $\rho$ is increasing and doubling to have

$$
\begin{aligned}
\sup _{0<R<3 r} \frac{\rho(R)}{|B(R)|} \int_{B(x, R)}|f(y)| d y & \lesssim \sup _{0<R<3 r} \frac{\rho(r)}{|B(x, R)|} \int_{B(x, R)}|f(y)| d y \\
& \leq \rho(r) M f(x) .
\end{aligned}
$$

For the second term, since $r \in(0, \infty) \mapsto r^{-n} \rho(r) \in(0, \infty)$ is decreasing and $f$ is supported on $B(x, r)$

$$
\begin{aligned}
\sup _{R \geq 3 r} \frac{\rho(R)}{|B(R)|} \int_{B(x, R)}|f(y)| d y & \leq \sup _{R \geq 3 r} \frac{\rho(3 r)}{|B(x, 3 r)|} \int_{B(x, R)}|f(y)| d y \\
& \leq \rho(r) M f(x) .
\end{aligned}
$$

Thus, combining these estimates, we obtain the desired result.

The Hedberg inequality for $M_{\rho}$ and $L^{\Phi}$ can be stated as follows:

Lemma 4.2. Let $\Phi, \Psi$ be Young functions. Assume that there exists a positive constant $C$ such that, for all $r>0$,

$$
\rho(r) \leq C \frac{\Psi^{-1}\left(r^{-n}\right)}{\Phi^{-1}\left(r^{-n}\right)} .
$$

Then, for any positive constant $C_{0}$, there exists a positive constant $C_{1}$ such that, for all $f \in L^{\Phi}\left(\mathbb{R}^{n}\right)$ with $f \neq 0$,

$$
M_{\rho} f(x) \leq C_{1}\|f\|_{L^{\Phi}}\left(\Psi^{-1} \circ \Phi\right)\left(\frac{M f(x)}{C_{0}\|f\|_{L^{\Phi}}}\right) \quad\left(x \in \mathbb{R}^{n}\right) .
$$


Proof. First note that

$$
\lim _{r \rightarrow \infty} \rho(r) \lesssim \frac{\Psi^{-1}(0)}{\Phi^{-1}(0)}
$$

if $\Phi^{-1}(0)>0$. Let $x \in \mathbb{R}^{n}$ be an arbitrary point. We may assume that $0<$ $M f(x)<\infty$ keeping in mind that $f$ does not vanish on a set of positive measure. Furthermore, we can assume that

$$
\Phi\left(\frac{M f(x)}{C_{0}\|f\|_{L^{\Phi}}}\right)<\infty
$$

otherwise there is nothing to do since $\Psi^{-1}(\infty)=\infty$. If

$$
\Phi\left(\frac{M f(x)}{C_{0}\|f\|_{L^{\Phi}}}\right)=0
$$

then

$$
\Phi^{-1}(0) \geq \frac{M f(x)}{C_{0}\|f\|_{L^{\Phi}}}>0
$$

according to the definition of $\Phi^{-1}$. Thus, thanks to (4.4)

$$
\begin{aligned}
\lim _{r \rightarrow \infty} \rho(r) & \lesssim \frac{\Psi^{-1}(0)}{\Phi^{-1}(0)} \\
& =\frac{1}{\Phi^{-1}(0)} \Psi^{-1} \circ \Phi\left(\frac{M f(x)}{C_{0}\|f\|_{L^{\Phi}}}\right) \\
& \leq \frac{C_{0}\|f\|_{L^{\Phi}}}{M f(x)} \Psi^{-1} \circ \Phi\left(\frac{M f(x)}{C_{0}\|f\|_{L^{\Phi}}}\right) .
\end{aligned}
$$

Thus by (4.1) we have

$$
M_{\rho} f(x) \lesssim \lim _{r \rightarrow \infty} \rho(r) M f(x) \lesssim\|f\|_{L^{\Phi} \Psi^{-1} \circ \Phi}\left(\frac{M f(x)}{C_{0}\|f\|_{L^{\Phi}}}\right) .
$$

It thus remains to handle the case where

$$
0<\Phi\left(\frac{M f(x)}{C_{0}\|f\|_{L^{\Phi}}}\right)<\infty .
$$

In the case we can choose $r>0$ such that

$$
r^{-n}=\Phi\left(\frac{M f(x)}{C_{0}\|f\|_{L^{\Phi}}}\right) .
$$

Let $B=B(x, r)$ and represent $f$ as

$$
f=f_{1}+f_{2}, \quad f_{1}=f \chi_{B}, \quad f_{2}=f \chi_{\mathbb{R}^{n} \backslash B}
$$


so that $M_{\rho} f(x) \leq M_{\rho} f_{1}(x)+M_{\rho} f_{2}(x)$.

We have (4.1) for $f_{1}$. Meanwhile by Lemma 2.7 .

$$
\begin{aligned}
M_{\rho} f_{2}(x) & =\sup _{t>0} \frac{\rho(t)}{|B(x, t)|} \int_{B(x, t) \cap \mathbb{R}^{n} \backslash B(x, r)}|f(z)| d z \\
& =\sup _{r<t<\infty} \frac{\rho(t)}{|B(x, t)|} \int_{B(x, t)}|f(z)| d z \\
& \lesssim \sup _{r<t<\infty} \rho(t) \Phi^{-1}\left(|B(x, t)|^{-1}\right)\|f\|_{L^{\Phi}(B(x, t))} \\
& \lesssim\|f\|_{L^{\Phi}} \sup _{r<t<\infty} \rho(t) \Phi^{-1}\left(t^{-n}\right)
\end{aligned}
$$

Consequently we have by Lemma 4.1

$$
M_{\rho} f(x) \lesssim \rho(r) M f(x)+\|f\|_{L^{\Phi}} \sup _{r<t<\infty} \rho(t) \Phi^{-1}\left(t^{-n}\right) .
$$

Thus, by (4.2) and the monotonicity of $\Psi^{-1}$ we obtain

$$
M_{\rho} f(x) \lesssim M f(x) \frac{\Psi^{-1}\left(r^{-n}\right)}{\Phi^{-1}\left(r^{-n}\right)}+\|f\|_{L^{\Phi}} \Psi^{-1}\left(r^{-n}\right) .
$$

Since $\Phi^{-1}\left(r^{-n}\right)=\frac{M f(x)}{C_{0}\|f\|_{L^{\Phi}}}$, we have

$$
M_{\rho} f(x) \lesssim\|f\|_{L^{\Phi}} \Psi^{-1}\left(r^{-n}\right)=\|f\|_{L^{\Phi}} \Psi^{-1}\left(\Phi\left(\frac{M f(x)}{C_{0}\|f\|_{L^{\Phi}}}\right)\right) .
$$

Therefore, we get (4.3).

In 10] we obtain a counterpart to generalized Orlicz-Morrey spaces of the second kind defined in [9]. However, as is written in [9] generalized Orlicz-Morrey spaces of the second kind do not cover $L^{2}\left(\mathbb{R}^{n}\right) \cap L^{3}\left(\mathbb{R}^{n}\right)$. So, the following theorem can be viewed as a different theorem from [9]:

Theorem 4.3. Let $\Phi, \Psi$ be Young functions. Assume that $\rho$ is increasing and that $r \mapsto r^{-n} \rho(r)$ is decreasing. Then the condition (4.2) is necessary and sufficient for the boundedness of $M_{\rho}$ from $L^{\Phi}\left(\mathbb{R}^{n}\right)$ to $\mathrm{WL} L^{\Psi}\left(\mathbb{R}^{n}\right)$. Moreover, if $\Phi \in \nabla_{2}$, then the condition (4.2) is necessary and sufficient for the boundedness of $M_{\rho}$ from $L^{\Phi}\left(\mathbb{R}^{n}\right)$ to $L^{\Psi}\left(\mathbb{R}^{n}\right)$.

Proof. We start with the necessity. For the necessity, we can concentrate on the boundedness of $M_{\rho}$ from $L^{\Phi}\left(\mathbb{R}^{n}\right)$ to $\mathrm{W} L^{\Psi}\left(\mathbb{R}^{n}\right)$, since the boundedness of $M_{\rho}$ from $L^{\Phi}\left(\mathbb{R}^{n}\right)$ to $L^{\Psi}\left(\mathbb{R}^{n}\right)$ is stronger than the boundedness of $M_{\rho}$ from $L^{\Phi}\left(\mathbb{R}^{n}\right)$ to $\mathrm{W} L^{\Psi}\left(\mathbb{R}^{n}\right)$. With this in mind, assume that $M_{\rho}$ is bounded from $L^{\Phi}\left(\mathbb{R}^{n}\right)$ to $\mathrm{W} L^{\Psi}\left(\mathbb{R}^{n}\right)$. We utilize a trivial pointwise estimate

$$
\rho(r) \chi_{B(0, r)} \leq M_{\rho} \chi_{B(0,2 r)} .
$$


Therefore, by the doubling property of $\Phi^{-1}$ and Lemma 2.6, we have

$$
\begin{aligned}
\rho(r) & \lesssim \Psi^{-1}\left(r^{-n}\right)\left\|M_{\rho} \chi_{B(0,2 r)}\right\|_{\mathrm{W} L^{\Psi}(B(0, r))} \\
& \lesssim \Psi^{-1}\left(r^{-n}\right)\left\|M_{\rho} \chi_{B(0,2 r)}\right\|_{\mathrm{W} L^{\Psi}} \\
& \lesssim \Psi^{-1}\left(r^{-n}\right)\left\|\chi_{B(0,2 r)}\right\|_{L^{\Phi}} \\
& \lesssim \frac{\Psi^{-1}\left(r^{-n}\right)}{\Phi^{-1}\left(r^{-n}\right)} .
\end{aligned}
$$

We move on to the sufficiency. Here and below we let $f$ be a nonzero measurable function.

- Let $C_{0}$ be as in (2.3). Then by (2.3) and (4.3), we have

$$
\begin{aligned}
& \sup _{r>0} \Psi(r) m\left(\frac{M_{\rho} f(y)}{C_{1}\|f\|_{L^{\Phi}}}, r\right)=\sup _{r>0} m\left(\Psi\left(\frac{M_{\rho} f(y)}{C_{1}\|f\|_{L^{\Phi}}}\right), r\right) \\
\leq & \sup _{r>0} r m\left(\Phi\left(\frac{M f(y)}{C_{0}\|f\|_{L^{\Phi}}}\right), r\right) \leq \sup _{r>0} \Phi(r) m\left(\frac{M f(y)}{\|M f\|_{\mathrm{W} L^{\Phi}}}, r\right) \leq 1,
\end{aligned}
$$

i.e.

$$
\left\|M_{\rho} f\right\|_{\mathrm{W} L^{\Psi}} \lesssim\|f\|_{L^{\Phi}} .
$$

- Assume in addition that $\Phi \in \nabla_{2}$. Let $C_{0}$ be as in (2.4). By (2.4) and (4.3), we have

$$
\begin{aligned}
\int_{\mathbb{R}^{n}} \Psi\left(\frac{M_{\rho} f(y)}{C_{1}\|f\|_{L^{\Phi}}}\right) d y & \leq \int_{\mathbb{R}^{n}} \Phi\left(\frac{M f(y)}{C_{0}\|f\|_{L^{\Phi}}}\right) d y \\
& \leq \int_{\mathbb{R}^{n}} \Phi\left(\frac{M f(y)}{\|M f\|_{L^{\Phi}}}\right) d y \leq 1
\end{aligned}
$$

i.e.

$$
\left\|M_{\rho} f\right\|_{L^{\Psi}} \lesssim\|f\|_{L^{\Phi}}
$$

\section{Generalized Orlicz-Morrey spaces of the third kind}

In [3], the generalized Orlicz-Morrey space $M^{\Phi, \varphi}\left(\mathbb{R}^{n}\right)$ was introduced to unify Orlicz spaces and generalized Morrey spaces. Other definitions of generalized OrliczMorrey spaces can be found in [22, 31]. In words of [6], our generalized OrliczMorrey space is the third kind and the ones in [22] and [31] are the first kind and the second kind, respectively. Notice that the definition of the space of the third kind relies only on the fact that $L^{\Phi}\left(\mathbb{R}^{n}\right)$ is a normed linear space, which is independent of the condition that it is generated by modulars.

The definition of generalized Orlicz-Morrey spaces of the third kind is as follows: 
Definition 5.1. Let $\varphi$ be a positive measurable function on $(0, \infty)$ and $\Phi$ any Young function. We denote by $\mathcal{M}^{\Phi, \varphi}\left(\mathbb{R}^{n}\right)$ the generalized Orlicz-Morrey space of the third kind, the space of all functions $f \in L_{\text {loc }}^{\Phi}\left(\mathbb{R}^{n}\right)$ with finite norm

$$
\|f\|_{\mathcal{M}^{\Phi, \varphi}}=\sup _{x \in \mathbb{R}^{n}, r>0} \varphi(r)^{-1} \Phi^{-1}\left(r^{-n}\right)\|f\|_{L^{\Phi}(B(x, r))} .
$$

Also by $\mathrm{WM}^{\Phi, \varphi}\left(\mathbb{R}^{n}\right)$ we denote the weak generalized Orlicz-Morrey space of the third kind of all measurable functions $f \in \mathrm{W} L_{\mathrm{loc}}^{\Phi}\left(\mathbb{R}^{n}\right)$ for which

$$
\|f\|_{\mathrm{W} \mathcal{M}^{\Phi, \varphi}}=\sup _{x \in \mathbb{R}^{n}, r>0} \varphi(r)^{-1} \Phi^{-1}\left(r^{-n}\right)\|f\|_{\mathrm{W} L^{\Phi}(B(x, r))}<\infty .
$$

A function $\varphi:(0, \infty) \rightarrow(0, \infty)$ is said to be almost increasing (resp. almost decreasing) if there exists a constant $C>0$ such that

$$
\varphi(r) \leq C \varphi(s) \quad(\text { resp. } \varphi(r) \geq C \varphi(s)) \quad \text { for } r \leq s .
$$

For a Young function $\Phi$, we denote by $\mathcal{G}_{\Phi}$ the set of all $\varphi:(0, \infty) \rightarrow(0, \infty)$ functions such that $t \in(0, \infty) \mapsto \frac{\varphi(t)}{\Phi^{-1}\left(t^{-n}\right)}$ is almost increasing and $t \in(0, \infty) \mapsto \frac{\varphi(t)}{\Phi^{-1}\left(t^{-n}\right) t^{n}}$ is almost decreasing. Note that $\varphi \in \mathcal{G}_{\Phi}$ implies doubling condition of $\varphi$.

We investigate the structure of $\mathcal{M}^{\Phi, \varphi}\left(\mathbb{R}^{n}\right)$. We denote by $\Theta$ the set of all measurable functions equivalent to 0 on $\mathbb{R}^{n}$. To exclude some trivial cases, we can use the following lemma was proved in [4]:

Lemma 5.2. Let $\Phi$ be a Young function and $\varphi$ be a positive measurable function on $(0, \infty)$.

(i) If

$$
\sup _{t<r<\infty} \frac{\Phi^{-1}\left(r^{-n}\right)}{\varphi(r)}=\infty \quad \text { for some } t>0
$$

then $\mathcal{M}^{\Phi, \varphi}\left(\mathbb{R}^{n}\right)=\Theta$.

(ii) If

$$
\sup _{0<r<\tau} \varphi(r)^{-1}=\infty \quad \text { for some } \tau>0
$$

then $\mathcal{M}^{\Phi, \varphi}\left(\mathbb{R}^{n}\right)=\Theta$.

Remark 5.3. If

$$
\sup _{0<r \leq t} \frac{\Phi^{-1}\left(r^{-n}\right) r^{n}}{\varphi(r)}=\infty \quad \text { for some } t>0,
$$

then $\mathcal{M}^{\Phi, \varphi}=\Theta$. Actually, by Remark 2.2 one has

$$
\Phi^{-1}\left(r^{-n}\right) r^{n} \leq \Phi^{-1}\left(t^{-n}\right) t^{n}<\infty
$$

and then

$$
\sup _{0<r<t} \varphi(r)^{-1}=\infty
$$


Remark 5.4. Based on Lemma 5.2 and Remark 5.3 and an observation similar to the one made by Nakai [17, p. 446] it can be assumed that $\varphi \in \mathcal{G}_{\Phi}$ in the definition of $\mathcal{M}^{\Phi, \varphi}\left(\mathbb{R}^{n}\right)$. More explicitly, we have the following observation:

(i) By Lemma 5.2 we may assume that $\inf _{r \leq t<\infty} \frac{\varphi(t)}{\Phi^{-1}\left(t^{-n}\right)}>0$ for every $r>0$. Let

$$
\psi(r)=\Phi^{-1}\left(r^{-n}\right) \inf _{r \leq t<\infty} \frac{\varphi(t)}{\Phi^{-1}\left(t^{-n}\right)}, \quad r>0 .
$$

Then $r \in(0, \infty) \mapsto \frac{\psi(r)}{\Phi^{-1}\left(r^{-n}\right)}$ is increasing and $\mathcal{M}^{\Phi, \varphi}\left(\mathbb{R}^{n}\right)=\mathcal{M}^{\Phi, \psi}\left(\mathbb{R}^{n}\right)$ with equivalent norms. Indeed, it is clear that $\psi(r) \leq \varphi(r)$ by the definition of $\psi$. Hence $\mathcal{M}^{\Phi, \psi}\left(\mathbb{R}^{n}\right) \subset \mathcal{M}^{\Phi, \varphi}\left(\mathbb{R}^{n}\right)$ and $\|f\|_{\mathcal{M}^{\Phi, \varphi}} \leq\|f\|_{\mathcal{M}^{\Phi, \psi}}$. On the other hand,

$$
\begin{aligned}
\sup _{r>0} \psi(r)^{-1} \Phi^{-1}\left(r^{-n}\right)\|f\|_{L^{\Phi}(B(x, r))} & =\sup _{r>0} \frac{1}{\inf _{r \leq t<\infty} \frac{\varphi(t)}{\Phi^{-1}\left(t^{-n}\right)}}\|f\|_{L^{\Phi}(B(x, r))} \\
& =\sup _{r>0}\left(\sup _{r \leq t<\infty} \frac{\Phi^{-1}\left(t^{-n}\right)}{\varphi(t)}\right)\|f\|_{L^{\Phi}(B(x, r))} \\
& \leq \sup _{t>0} \varphi(t)^{-1} \Phi^{-1}\left(t^{-n}\right)\|f\|_{L^{\Phi}(B(x, t))} .
\end{aligned}
$$

Hence $\mathcal{M}^{\Phi, \varphi}\left(\mathbb{R}^{n}\right) \subset \mathcal{M}^{\Phi, \psi}\left(\mathbb{R}^{n}\right)$ and $\|f\|_{\mathcal{M}^{\Phi, \psi}} \leq\|f\|_{\mathcal{M}^{\Phi, \varphi}}$.

(ii) By Remark 5.3 we may assume that $\inf _{0<t \leq r} \frac{\varphi(t)}{\Phi^{-1}\left(t^{-n}\right) t^{n}}>0$ for every $r>0$. Define $\psi(r)$ by the formula:

$$
\sup _{t \in(0, r]} \frac{\Phi^{-1}\left(t^{-n}\right) t^{n}}{\varphi(t)}=\frac{\Phi^{-1}\left(r^{-n}\right) r^{n}}{\psi(r)} .
$$

It is easy to see that $\psi(r) \leq \varphi(r)$ for any $r>0$. Thus, $\mathcal{M}^{\Phi, \psi}\left(\mathbb{R}^{n}\right) \subset \mathcal{M}^{\Phi, \varphi}\left(\mathbb{R}^{n}\right)$ and $\|f\|_{\mathcal{M}^{\Phi, \varphi}} \leq\|f\|_{\mathcal{M}^{\Phi, \psi}}$. Conversely, let $f \in \mathcal{M}^{\Phi, \varphi}\left(\mathbb{R}^{n}\right)$. For any $r \in(0, \infty)$, choose $t \in(0, r]$ so that

$$
\frac{\Phi^{-1}\left(r^{-n}\right) r^{n}}{\psi(r)} \leq 2 \frac{\Phi^{-1}\left(t^{-n}\right) t^{n}}{\varphi(t)}
$$

and cover $B(x, r)$ with a family of $N$ balls $\left\{B\left(x_{j}, t\right)\right\}_{j=1}^{N}$, where $N \lesssim r^{-n} t^{n}$. Let $j_{0}$ be such that

$$
\begin{aligned}
\|f\|_{L^{\Phi}(B(x, r))} & \leq N\|f\|_{L^{\Phi}\left(B\left(x_{j_{0}}, t\right)\right)} \\
& =N \max _{j=1,2 \ldots, N}\|f\|_{L^{\Phi}\left(B\left(x_{j}, t\right)\right)} \\
& \lesssim t^{-n} r^{n}\|f\|_{L^{\Phi}\left(B\left(x_{j_{0}}, r\right)\right)}
\end{aligned}
$$


Thus,

$$
\begin{aligned}
\frac{\Phi^{-1}\left(r^{-n}\right)}{\psi(r)}\|f\|_{L^{\Phi}(B(x, r))} & \lesssim \frac{r^{n} \Phi^{-1}\left(r^{-n}\right)}{\psi(r) t^{n}}\|f\|_{L^{\Phi}\left(B\left(x_{j_{0}}, t\right)\right)} \\
& \leq 2 \frac{\Phi^{-1}\left(t^{-n}\right)}{\varphi(t)}\|f\|_{L^{\Phi}\left(B\left(x_{j_{0}}, t\right)\right)} \\
& \leq 2\|f\|_{\mathcal{M}^{\Phi, \varphi},}
\end{aligned}
$$

implying $f \in \mathcal{M}^{\Phi, \varphi}\left(\mathbb{R}^{n}\right)$ and $\|f\|_{\mathcal{M}^{\Phi, \psi}} \leq\|f\|_{\mathcal{M}^{\Phi, \varphi}}$. Thus, $\mathcal{M}^{\Phi, \psi}\left(\mathbb{R}^{n}\right) \hookrightarrow \mathcal{M}^{\Phi, \varphi}\left(\mathbb{R}^{n}\right)$.

As the following lemma shows, $\mathcal{G}_{\Phi}$ is useful:

Lemma $5.5([5])$. Let $B_{0}:=B\left(x_{0}, r_{0}\right)$. If $\varphi \in \mathcal{G}_{\Phi}$ is almost decreasing, then there exist $C>0$ such that

$$
\frac{1}{\varphi\left(r_{0}\right)} \leq\left\|\chi_{B_{0}}\right\|_{\mathcal{M}^{\Phi, \varphi}} \leq \frac{C}{\varphi\left(r_{0}\right)} .
$$

\section{Generalized fractional integrals on generalized Orlicz-Morrey spaces}

We remark that there are two types of the boundedness of the fractional integral operators. One is the Spanne-type boundedness obtained in [28]. Another boundedness is of Adams-type obtained by Adams [1. In the classical case due to the fact that Morrey spaces are nested we can say that the Adams-type boundedness is stronger than the Spanne-type boundedness. However, we need to depend on the pointwise estimate of Hedberg-type [12], so the Adams-type boundedness is unavailable for local Morrey spaces. In this section we give a characterization for the Spanne-type boundedness and the Adams-type boundedness of the operator $I_{\rho}$ on generalized Orlicz-Morrey spaces, respectively.

\subsection{Spanne-type result}

We need the following lemma is valid:

Lemma 6.1. Let $\Phi, \Psi$ be Young functions, and let $\rho$ satisfy (1.1) and (1.4). Assume that the condition (3.1) is fulfilled. Then there exists a positive constant $C$ such that, for all $f \in L_{\mathrm{loc}}^{\Phi}\left(\mathbb{R}^{n}\right)$ and $B=B(x, r)$,

$$
\begin{aligned}
& \left\|I_{\rho} f\right\|_{\mathrm{W} L^{\Psi}(B)} \\
& \leq C\|f\|_{L^{\Phi}(2 B)}+\frac{C}{\Psi^{-1}\left(r^{-n}\right)} \int_{2 k_{1} r}^{\infty}\|f\|_{L^{\Phi}(B(x, t))} \Phi^{-1}\left(t^{-n}\right) \rho(t) \frac{d t}{t} .
\end{aligned}
$$


Moreover if we assume $\Phi \in \nabla_{2}$, the following inequality is also valid:

$$
\begin{aligned}
& \left\|I_{\rho} f\right\|_{L^{\Psi}(B)} \\
& \leq C\|f\|_{L^{\Phi}(2 B)}+\frac{C}{\Psi^{-1}\left(r^{-n}\right)} \int_{2 k_{1} r}^{\infty}\|f\|_{L^{\Phi}(B(x, t))} \Phi^{-1}\left(t^{-n}\right) \rho(t) \frac{d t}{t} .
\end{aligned}
$$

Proof. We represent $f$ as

$$
f=f_{1}+f_{2}, \quad f_{1}=f \chi_{2 B}, \quad f_{2}=f-f_{1}, \quad r>0 .
$$

Then we have

$$
\left\|I_{\rho} f\right\|_{W L^{\Psi}(B)} \leq 2\left(\left\|I_{\rho} f_{1}\right\|_{W L^{\Psi}(B)}+\left\|I_{\rho} f_{2}\right\|_{W L^{\Psi}(B)}\right) .
$$

From the boundedness of $I_{\rho}$ from $L^{\Phi}\left(\mathbb{R}^{n}\right)$ to $W L^{\Psi}\left(\mathbb{R}^{n}\right)$ (see Theorem 3.1) it follows that:

$$
\left\|I_{\rho} f_{1}\right\|_{W L^{\Psi}(B)} \lesssim\left\|I_{\rho} f_{1}\right\|_{W L^{\Psi}\left(\mathbb{R}^{n}\right)} \leq C\left\|f_{1}\right\|_{L^{\Phi}\left(\mathbb{R}^{n}\right)}=C\|f\|_{L^{\Phi}(2 B)},
$$

where constant $C>0$ is independent of $f$.

For $f_{2}$ we have

$$
\left|I_{\rho} f_{2}(y)\right| \leq \int_{\mathrm{C}_{(2 B)}} \frac{\rho(|y-z|)}{|y-z|^{n}}|f(z)| d z=\sum_{j=1}^{\infty} \int_{2^{j+1} B \backslash 2^{j} B} \frac{\rho(|y-z|)}{|y-z|^{n}}|f(z)| d z .
$$

A geometric observation shows that $y \in B, z \in \mathbb{R}^{n} \backslash(2 B)$ implies

$$
\frac{1}{2}|x-z| \leq|y-z| \leq \frac{3}{2}|x-z| .
$$

Using (1.4) and Lemma 2.7, we have

$$
\begin{aligned}
& \int_{2^{j+1} B \backslash 2^{j} B} \frac{\rho(|y-z|)}{|y-z|^{n}}|f(z)| d z \\
& \left.\lesssim \int_{2^{j-1} r \leq t \leq 3 \cdot 2^{j} r} \rho(t)\right) \frac{1}{\left|2^{j} B\right|} \int_{2^{j+1} B \backslash 2^{j} B}|f(z)| d z \\
& \lesssim \int_{2^{j} k_{1} r}^{3 \cdot 2^{j} k_{2} r} \frac{\rho(t)}{t} d t\|f\|_{L^{\Phi}\left(B\left(x, 2^{j+1} r\right)\right)} \Phi^{-1}\left(\left|2^{j+1} B\right|^{-1}\right) \\
& \lesssim \int_{2^{j} k_{1} r}^{2^{j} \max \left(3 k_{2}, 4\right) r}\|f\|_{L^{\Phi}(B(x, t))} \Phi^{-1}\left(t^{-n}\right) \frac{\rho(t)}{t} d t .
\end{aligned}
$$

Then

$$
\left|I_{\rho} f_{2}(y)\right| \lesssim \int_{2 k_{1} r}^{\infty}\|f\|_{L^{\Phi}(B(x, t))} \Phi^{-1}\left(t^{-n}\right) \frac{\rho(t)}{t} d t
$$


Thus by Lemma 2.6 we have

$$
\left\|I_{\rho} f_{2}\right\|_{W L^{\Psi}(B)} \lesssim \frac{1}{\Psi^{-1}\left(r^{-n}\right)} \int_{2 k_{1} r}^{\infty}\|f\|_{L^{\Phi}(B(x, t))} \Phi^{-1}\left(t^{-n}\right) \rho(t) \frac{d t}{t} .
$$

Therefore we obtain (6.1) by (6.3) and (6.5).

If $\Phi \in \nabla_{2}$, then we can use strong type inequality instead of (6.3) and obtain (6.2) by using the same argument.

Remark 6.2. In the case $\Phi(t)=t^{p}(1 \leq p<\infty)$ Lemma 6.1] was proved in [7].

The following theorem gives necessary and sufficient conditions for Spanne-type boundedness of the operator $I_{\rho}$ from $\mathcal{M}^{\Phi, \varphi_{1}}\left(\mathbb{R}^{n}\right)$ to $\mathcal{M}^{\Psi, \varphi_{2}}\left(\mathbb{R}^{n}\right)$.

Theorem 6.3 (Spanne-type result). Let $\Phi, \Psi$ be Young functions, and let $\varphi_{1} \in \mathcal{G}_{\Phi}$ and $\varphi_{2} \in \mathcal{G}_{\Psi}$.

1. Let $\rho$ satisfy (1.1) and (1.4). Assume that (3.1) is fulfilled. Then the conditions

$$
\begin{gathered}
\frac{\varphi_{1}(r)}{\Phi^{-1}\left(r^{-n}\right)} \leq C \frac{\varphi_{2}(r)}{\Psi^{-1}\left(r^{-n}\right)}, \\
\int_{r}^{\infty} \varphi_{1}(t) \rho(t) \frac{d t}{t} \leq C \varphi_{2}(r),
\end{gathered}
$$

for all $r>0$, where $C>0$ does not depend on $r$, are sufficient for the boundedness of $I_{\rho}$ from $\mathcal{M}^{\Phi, \varphi_{1}}\left(\mathbb{R}^{n}\right)$ to $\mathrm{WM}^{1, \varphi_{2}}\left(\mathbb{R}^{n}\right)$. Moreover, if $\Phi \in \nabla_{2}$, then the conditions (6.6) and (6.7) are sufficient for the boundedness of $I_{\rho}$ from $\mathcal{M}^{\Phi, \varphi_{1}}\left(\mathbb{R}^{n}\right)$ to $\mathcal{M}^{\Psi, \varphi_{2}}\left(\mathbb{R}^{n}\right)$.

2. Let $\varphi_{1}$ be almost decreasing. Then the condition

$$
\varphi_{1}(r) \int_{0}^{r} \frac{\rho(t)}{t} d t \leq C \varphi_{2}(r)
$$

for all $r>0$, where $C>0$ does not depend on $r$, is necessary for the boundedness of $I_{\rho}$ from $\mathcal{M}^{\Phi, \varphi_{1}}\left(\mathbb{R}^{n}\right)$ to $\mathrm{WM}^{1, \varphi_{2}}\left(\mathbb{R}^{n}\right)$ and hence $\mathcal{M}^{\Phi, \varphi_{1}}\left(\mathbb{R}^{n}\right)$ to $\mathcal{M}^{\Psi, \varphi_{2}}\left(\mathbb{R}^{n}\right)$.

3. Let $\rho$ satisfy (1.1) and (1.4). Assume that (3.1) is fulfilled, that $\varphi_{1}$ is almost decreasing and that $\varphi_{1}$ and $\varphi_{2}$ satisfy (6.6). Assume also that $\varphi_{1}$ and $\rho$ satisfy the condition

$$
\int_{r}^{\infty} \varphi_{1}(t) \rho(t) \frac{d t}{t} \leq C \varphi_{1}(r) \rho(r),
$$

for all $r>0$, where $C>0$ does not depend on $r$. Then the condition (6.8) is necessary and sufficient for the boundedness of $I_{\rho}$ from $\mathcal{M}^{\Phi, \varphi_{1}}\left(\mathbb{R}^{n}\right)$ to $\mathrm{WM}^{\Psi, \varphi_{2}}\left(\mathbb{R}^{n}\right)$. Moreover, if $\Phi \in \nabla_{2}$, then the condition (6.8) is necessary and sufficient for the boundedness of $I_{\rho}$ from $\mathcal{M}^{\Phi, \varphi_{1}}\left(\mathbb{R}^{n}\right)$ to $\mathcal{M}^{\Psi, \varphi_{2}}\left(\mathbb{R}^{n}\right)$. 
Proof. 1. By (6.1), (6.6) and (6.7) we have

$$
\begin{aligned}
\left\|I_{\rho} f\right\|_{\mathrm{WM}^{\Psi, \varphi_{2}}} \lesssim & \sup _{x \in \mathbb{R}^{n}, r>0} \varphi_{2}(r)^{-1} \Psi^{-1}\left(r^{-n}\right)\|f\|_{L^{\Phi}(B(x, 2 r))} \\
& +\sup _{x \in \mathbb{R}^{n}, r>0} \varphi_{2}(r)^{-1} \int_{2 k_{1} r}^{\infty}\|f\|_{L^{\Phi}(B(x, t))} \Phi^{-1}\left(t^{-n}\right) \rho(t) \frac{d t}{t} \\
\lesssim & \sup _{x \in \mathbb{R}^{n}, r>0} \varphi_{1}(r)^{-1} \Phi^{-1}\left(r^{-n}\right)\|f\|_{L^{\Phi}(B(x, r))} \\
& +\sup _{x \in \mathbb{R}^{n}, r>0} \varphi_{2}(r)^{-1} \int_{2 k_{1} r}^{\infty} \varphi_{1}(t)^{-1} \rho(t) \frac{d t}{t}\|f\|_{\mathcal{M}^{\Phi, \varphi_{1}}} \\
\lesssim & \|f\|_{\mathcal{M}^{\Phi, \varphi_{1}}} .
\end{aligned}
$$

Simply replace $\mathrm{W} L^{\Psi}(B)$ with $L^{\Psi}(B)$ and $\mathrm{W} \mathcal{M}^{\Psi, \eta}\left(\mathbb{R}^{n}\right)$ with $\mathcal{M}^{\Psi, \eta}\left(\mathbb{R}^{n}\right)$ and use (6.2), (6.6) and (6.7) for the strong estimate.

2. We will now prove the second part. Let $B_{R}=B(0, R)$ and $x \in B_{R / 2}$. By Lemma 3.3 we have

$$
\rho^{*}(R / 2):=\int_{0}^{R / 2} \frac{\rho(t)}{t} d t \leq C I_{\rho} \chi_{B_{R}}(x) .
$$

Therefore, by Lemma 5.5 and the doubling property of $\varphi_{1}$,

$$
\begin{aligned}
\rho^{*}(R / 2) & \lesssim\left|B_{R / 2}\right|^{-1}\left\|I_{\rho} \chi_{B_{R}}\right\|_{W L^{1}\left(B_{R / 2}\right)} \lesssim \varphi_{2}(R / 2)\left\|I_{\rho} \chi_{B_{R}}\right\|_{\mathrm{WM}^{1, \varphi_{2}}} \\
& \lesssim \varphi_{2}(R / 2)\left\|\chi_{B_{0}}\right\|_{\mathcal{M}^{\Phi, \varphi_{1}}} \lesssim \frac{\varphi_{2}(R / 2)}{\varphi_{1}(R)} \lesssim \frac{\varphi_{2}(R / 2)}{\varphi_{1}(R / 2)}
\end{aligned}
$$

Since this is true for every $R>0$, we are done.

3. The third statement of the theorem follows from the first and second parts of the theorem.

\subsection{Adams-type result}

The following theorem was proved in [3, Theorem 4.6]:

\section{Theorem 6.4.}

1. Let $\varphi \in \mathcal{G}_{\Phi}$ be almost decreasing. Then the maximal operator $M$ is bounded from $\mathcal{M}^{\Phi, \varphi}\left(\mathbb{R}^{n}\right)$ to $\mathrm{W} \mathcal{M}^{\Phi, \varphi}\left(\mathbb{R}^{n}\right)$.

2. Let $\Phi \in \nabla_{2}$ and $\varphi \in \mathcal{G}_{\Phi}$ be almost decreasing. Then the maximal operator $M$ is bounded on $\mathcal{M}^{\Phi, \varphi}\left(\mathbb{R}^{n}\right)$.

The following theorem gives necessary and sufficient conditions for Adams-type boundedness of the operator $I_{\rho}$ from $\mathcal{M}^{\Phi, \varphi}\left(\mathbb{R}^{n}\right)$ to $\mathcal{M}^{\Psi, \eta}\left(\mathbb{R}^{n}\right)$ : 
Theorem 6.5 (Adams-type result). Let $\Phi$ be a Yougn function, and let $\varphi \in \mathcal{G}_{\Phi}$ be almost decreasing. Let $\beta \in(0,1)$ and define $\eta(t) \equiv \varphi(t)^{\beta}$ for $t>0$ and $\Psi(t) \equiv$ $\Phi\left(t^{1 / \beta}\right)$ for $t>0$.

1. Let $\rho$ satisfy (1.1) and (1.4). Then the condition

$$
\varphi(r) \int_{0}^{r} \frac{\rho(t)}{t} d t+\int_{r}^{\infty} \rho(t) \varphi(t) \frac{d t}{t} \leq C \eta(r),
$$

for all $r>0$, where $C>0$ does not depend on $r$, is sufficient for the boundedness of $I_{\rho}$ from $\mathcal{M}^{\Phi, \varphi}\left(\mathbb{R}^{n}\right)$ to $\mathrm{WM}^{\Psi, \eta}\left(\mathbb{R}^{n}\right)$. Moreover, if $\Phi \in \nabla_{2}$, then the condition (6.10) is sufficient for the boundedness of $I_{\rho}$ from $\mathcal{M}^{\Phi, \varphi}\left(\mathbb{R}^{n}\right)$ to $\mathcal{M}^{\Psi, \eta}\left(\mathbb{R}^{n}\right)$.

2. The condition

$$
\varphi(r) \int_{0}^{r} \frac{\rho(t)}{t} d t \leq C \eta(r)
$$

for all $r>0$, where $C>0$ does not depend on $r$, is necessary for the boundedness of $I_{\rho}$ from $\mathcal{M}^{\Phi, \varphi}\left(\mathbb{R}^{n}\right)$ to $\mathrm{WM}^{1, \eta}\left(\mathbb{R}^{n}\right)$ and hence for the boundedness of $I_{\rho}$ from $\mathcal{M}^{\Phi, \varphi}\left(\mathbb{R}^{n}\right)$ to $\mathcal{M}^{\Psi, \eta}\left(\mathbb{R}^{n}\right)$.

3. Let $\rho$ satisfy (1.1) and (1.4). Assume that $\varphi$ satisfies the condition

$$
\int_{r}^{\infty} \rho(t) \varphi(t) \frac{d t}{t} \leq C \rho(r) \varphi(r)
$$

for all $r>0$, where $C>0$ does not depend on $r$. Then the condition (6.11) is necessary and sufficient for the boundedness of $I_{\rho}$ from $\mathcal{M}^{\Phi, \varphi}\left(\mathbb{R}^{n}\right)$ to $\mathrm{WM}^{\Psi, \eta}\left(\mathbb{R}^{n}\right)$. Moreover, if $\Phi \in \nabla_{2}$, then the condition (6.11) is necessary and sufficient for the boundedness of $I_{\rho}$ from $\mathcal{M}^{\Phi, \varphi}\left(\mathbb{R}^{n}\right)$ to $\mathcal{M}^{\Psi, \eta}\left(\mathbb{R}^{n}\right)$.

We notice that the function $\varphi$ and $\eta$ come into play, unlike Spanne-type. Similar to Lemma 4.2, we have the following pointwise estimate:

Lemma 6.6. Let $\Phi$ be a Young function, $\varphi \in \mathcal{G}_{\Phi}, \beta \in(0,1), \eta(t) \equiv \varphi(t)^{\beta}$ and $\Psi(t) \equiv \Phi\left(t^{1 / \beta}\right)$. If (6.10) holds, then there exists a positive constant $C$ such that, for all non-negative measurable functions $f$ and for every $x \in \mathbb{R}^{n}$,

$$
I_{\rho} f(x) \leq C(M f(x))^{\beta}\|f\|_{\mathcal{M}^{\Phi, \varphi}}^{1-\beta}
$$

Proof. Let $\tilde{\rho}$ be defined by (3.4). We have

$$
I_{\rho} f(x) \leq C\left[\sum_{j=-\infty}^{-1}+\sum_{j=0}^{\infty} \frac{\tilde{\rho}\left(2^{j} r\right)}{\left(2^{j} r\right)^{n}} \int_{|x-y|<2^{j} r} f(y) d y\right]=C(\mathrm{I}+\mathrm{II})
$$


for given $x \in \mathbb{R}^{n}$ and $r>0$. Thus from (3.5) and (3.6) with $\tau=\varphi$ we deduce

$$
\begin{aligned}
& \mathrm{I} \leq C \sum_{j=-\infty}^{-1} \tilde{\rho}\left(2^{j} r\right) M f(x) \leq C\left(\int_{0}^{k_{2} r} \frac{\rho(s)}{s} d s\right) M f(x) \\
& \mathrm{II} \leq C \sum_{j=0}^{\infty} \tilde{\rho}\left(2^{j} r\right) \varphi\left(2^{j} r\right)\|f\|_{\mathcal{M}^{\Phi, \varphi}} \leq C\|f\|_{\mathcal{M}^{\Phi, \varphi}} \int_{k_{1} r}^{\infty} \varphi(s) \frac{\rho(s)}{s} d s .
\end{aligned}
$$

Consequently we have

$$
I_{\rho} f(x) \lesssim\left(\int_{0}^{k_{2} r} \frac{\rho(s)}{s} d s\right) M f(x)+\|f\|_{\mathcal{M}^{\Phi, \varphi}} \int_{k_{1} r}^{\infty} \varphi(s) \frac{\rho(s)}{s} d s .
$$

Thus, the technique in [30, p. 6492] by (6.10) and the doubling property of $\varphi$ we obtain

$$
\begin{aligned}
I_{\rho} f(x) & \lesssim \min \left\{\varphi(r)^{\beta-1} M f(x), \varphi(r)^{\beta}\|f\|_{\mathcal{M}^{\Phi, \varphi}}\right\} \\
& \lesssim \sup _{s>0} \min \left\{s^{\beta-1} M f(x), s^{\beta}\|f\|_{\mathcal{M}^{\Phi, \varphi}}\right\} \\
& =(M f(x))^{\beta}\|f\|_{\mathcal{M}^{\Phi, \varphi}}^{1-\beta},
\end{aligned}
$$

where we have used that the supremum is achieved when the minimum parts are balanced. Hence we have $I_{\rho} f(x) \lesssim(M f(x))^{\beta}\|f\|_{\mathcal{M}^{\Phi, \varphi}}^{1-\beta}$.

We have the following scaling law:

Lemma 6.7. Let $\beta>0$. Let $\Psi$ and $\Phi$ be Yougn functions, and let $B$ be a ball. Then $\left\||f|^{\beta}\right\|_{L^{\Psi}(B)}=\|f\|_{L^{\Phi}(B)}^{\beta}$ and $\left\||f|^{\beta}\right\|_{\mathrm{W}_{L^{\Psi}(B)}}=\|f\|_{\mathrm{W} L^{\Phi}(B)}^{\beta}$ for all measurable functions $f$.

Proof. Simply note that

$$
\int_{B} \Psi\left(\frac{|f(x)|^{\beta}}{\|f\|_{L^{\Phi}(B)}^{\beta}}\right) d x=\int_{B} \Phi\left(\frac{|f(x)|}{\|f\|_{L^{\Phi}(B)}}\right) d x
$$

for $L^{\Phi}(B)$. The equality for weak spaces can be proved similarly.

\section{Proof of Theorem 6.5. 1.}

- We deal with the weak-type estimate. By using inequality (6.13) we have for an arbitrary ball $B$

$$
\left\|I_{\rho} f\right\|_{\mathrm{W} L^{\Psi}(B)} \lesssim\left\|(M f)^{\beta}\right\|_{\mathrm{W} L^{\Psi}(B)}\|f\|_{\mathcal{M}^{\Phi, \varphi}}^{1-\beta} .
$$

Consequently by using this inequality and Lemma 6.7 we have

$$
\left\|I_{\rho} f\right\|_{\mathrm{W} L^{\Psi}(B)} \lesssim\|M f\|_{\mathrm{W} L^{\Phi}(B)}^{\beta}\|f\|_{\mathcal{M}^{\Phi, \varphi}}^{1-\beta} .
$$


From Theorem 6.4 and (6.14), we get

$$
\begin{aligned}
\left\|I_{\rho} f\right\|_{\mathrm{W} \mathcal{M}^{\Psi, \eta},} & =\sup _{B} \eta(r)^{-1} \Psi^{-1}\left(r^{-n}\right)\left\|I_{\rho} f\right\|_{\mathrm{W} L^{\Psi}(B)} \\
& \lesssim\|f\|_{\mathcal{M}^{\Phi, \varphi}}^{1-\beta} \sup _{B} \eta(r)^{-1} \Psi^{-1}\left(r^{-n}\right)\|M f\|_{\mathrm{W} L^{\Phi}(B)}^{\beta} \\
& =\|f\|_{\mathcal{M}^{\Phi, \varphi}}^{1-\beta}\left(\sup _{B} \varphi(r)^{-1} \Phi^{-1}\left(r^{-n}\right)\|M f\|_{\mathrm{W} L^{\Phi}(B)}\right)^{\beta} \\
& \lesssim\|f\|_{\mathcal{M}^{\Phi, \varphi}} .
\end{aligned}
$$

- Simply replace $\mathrm{W} L^{\Psi}(B)$ with $L^{\Psi}(B)$ and $\mathrm{W} \mathcal{M}^{\Psi, \eta}\left(\mathbb{R}^{n}\right)$ with $\mathcal{M}^{\Psi, \eta}\left(\mathbb{R}^{n}\right)$ for the strong estimate.

2. We will now prove the second part. Let $B_{R}=B(0, R)$ and $x \in B_{R / 2}$. By Lemmas 2.6, 3.3 and 5.5 and the doubling property of $\varphi$, we have

$$
\begin{aligned}
\rho^{*}(R / 2) & \leq C\left|B_{R / 2}\right|^{-1}\left\|I_{\rho} \chi_{B_{R}}\right\|_{\mathrm{W} L^{1}\left(B_{R / 2}\right)} \leq C \eta(R / 2)\left\|I_{\rho} \chi_{B_{R}}\right\|_{\mathrm{WM}^{1, \eta}} \\
& \leq C \eta(R / 2)\left\|\chi_{B_{R}}\right\|_{\mathcal{M}^{\Phi, \varphi}} \leq C \frac{\eta(R / 2)}{\varphi(R)} \leq C \frac{\eta(R / 2)}{\varphi(R / 2)}=C \varphi(R / 2)^{\beta-1} .
\end{aligned}
$$

Since this is true for every $R>0$, the proof is complete.

3. This part follows from the first and second parts.

\section{Generalized fractional maximal operators on generalized Orlicz-Morrey spaces}

In this section we give a characterization for the Spanne-type boundedness and the Adams-type boundedness of the operator $M_{\rho}$ on generalized Orlicz-Morrey spaces, respectively.

\subsection{Spanne-type result}

We use the following lemma:

Lemma 7.1. Let $\Phi, \Psi$ be Young functions. Assume that $\rho$ is increasing and that $r \mapsto r^{-n} \rho(r)$ is decreasing. Assume also that the condition (4.2) is fulfilled. Then there exists a positive constant $C$ such that, for all $f \in L_{\mathrm{loc}}^{\Phi}\left(\mathbb{R}^{n}\right)$ and $B=B(x, r)$,

$$
\begin{aligned}
& \left\|M_{\rho} f\right\|_{\mathrm{W} L^{\Psi}(B)} \\
& \leq C\|f\|_{L^{\Phi}(2 B)}+\frac{C}{\Psi^{-1}\left(r^{-n}\right)} \sup _{r<t<\infty}\|f\|_{L^{\Phi}(B(x, 2 t))} \Phi^{-1}\left(t^{-n}\right) \rho(t) .
\end{aligned}
$$


Moreover if we assume $\Phi \in \nabla_{2}$, the following inequality is also valid:

$$
\begin{aligned}
& \left\|M_{\rho} f\right\|_{L^{\Psi}(B)} \\
& \leq C\|f\|_{L^{\Phi}(2 B)}+\frac{C}{\Psi^{-1}\left(r^{-n}\right)} \sup _{r<t<\infty}\|f\|_{L^{\Phi}(B(x, 2 t))} \Phi^{-1}\left(t^{-n}\right) \rho(t) .
\end{aligned}
$$

Proof. We represent $f$ as

$$
f=f_{1}+f_{2}, \quad f_{1}=f \chi_{2 B}, \quad f_{2}=f-f_{1}, \quad r>0 .
$$

Then we have

$$
\left\|M_{\rho} f\right\|_{W L^{\Psi}(B)} \lesssim\left\|M_{\rho} f_{1}\right\|_{W L^{\Psi}(B)}+\left\|M_{\rho} f_{2}\right\|_{W L^{\Psi}(B)} .
$$

From the boundedness of $M_{\rho}$ from $L^{\Phi}\left(\mathbb{R}^{n}\right)$ to $W L^{\Psi}\left(\mathbb{R}^{n}\right)$ (see Theorem 4.3) it follows that:

$$
\left\|M_{\rho} f_{1}\right\|_{W L^{\Psi}(B)} \leq\left\|M_{\rho} f_{1}\right\|_{W L^{\Psi}\left(\mathbb{R}^{n}\right)} \leq C\left\|f_{1}\right\|_{L^{\Phi}\left(\mathbb{R}^{n}\right)}=C\|f\|_{L^{\Phi}(2 B)},
$$

where constant $C>0$ is independent of $f$.

If $y \in B$ and $r<t$, then $B(y, t) \subset B(x, 2 t)$. Then, using Lemma 2.7, we have

$$
\begin{aligned}
M_{\rho} f_{2}(y) & =\sup _{t>0} \frac{\rho(t)}{|B(y, t)|} \int_{B(y, t) \backslash(B(x, 2 r))}|f(z)| d z \\
& \leq \sup _{t>r} \frac{\rho(t)}{|B(x, t)|} \int_{B(x, 2 t)}|f(z)| d z \\
& \lesssim \sup _{r<t<\infty} \rho(t) \Phi^{-1}\left(t^{-n}\right)\|f\|_{L^{\Phi}(B(x, 2 t))} \quad \text { for } y \in B .
\end{aligned}
$$

Thus by Lemma 2.6 we have

$$
\left\|M_{\rho} f_{2}\right\|_{W L^{\Psi}(B)} \lesssim \frac{1}{\Psi^{-1}\left(r^{-n}\right)} \sup _{r<t<\infty} \rho(t) \Phi^{-1}\left(t^{-n}\right)\|f\|_{L^{\Phi}(B(x, 2 t))} .
$$

Therefore we obtain (77.1) by (7.3) and (7.5)

If $\Phi \in \nabla_{2}$, then we can use strong type inequality instead of (7.3) and obtain (7.2) by using the same argument.

The following theorem gives a necessary and sufficient condition for Spanne-type boundedness of the operator $M_{\rho}$ from $\mathcal{M}^{\Phi, \varphi_{1}}\left(\mathbb{R}^{n}\right)$ to $\mathcal{M}^{\Psi, \varphi_{2}}\left(\mathbb{R}^{n}\right)$ : We notice that the requirement is the same as the Orlicz spaces.

Theorem 7.2 (Spanne-type result). Let $\Phi, \Psi$ be Young functions, and let $\varphi_{1} \in \mathcal{G}_{\Phi}$ and $\varphi_{2} \in \mathcal{G}_{\Psi}$. 
1. Assume that $\rho$ is increasing and that $r \mapsto r^{-n} \rho(r)$ is decreasing. Assume also that the conditions (4.2) and (6.6) are satisfied. Then the condition

$$
\sup _{r<t<\infty} \varphi_{1}(t) \rho(t) \leq C \varphi_{2}(r)
$$

for all $r>0$, where $C>0$ does not depend on $r$, are sufficient for the boundedness of $M_{\rho}$ from $\mathcal{M}^{\Phi, \varphi_{1}}\left(\mathbb{R}^{n}\right)$ to $\mathrm{W} \mathcal{M}^{\Psi, \varphi_{2}}\left(\mathbb{R}^{n}\right)$. Moreover, if $\Phi \in \nabla_{2}$, then the condition (7.6) is sufficient for the boundedness of $M_{\rho}$ from $\mathcal{M}^{\Phi, \varphi_{1}}\left(\mathbb{R}^{n}\right)$ to $\mathcal{M}^{\Psi, \varphi_{2}}\left(\mathbb{R}^{n}\right)$.

2. Let $\varphi_{1}$ be almost decreasing. Then the condition

$$
\varphi_{1}(r) \rho(r) \leq C \varphi_{2}(r),
$$

for all $r>0$, where $C>0$ does not depend on $r$, is necessary for the boundedness of $M_{\rho}$ from $\mathcal{M}^{\Phi, \varphi_{1}}\left(\mathbb{R}^{n}\right)$ to $\mathrm{W} \mathcal{M}^{1, \varphi_{2}}\left(\mathbb{R}^{n}\right)$ and hence $\mathcal{M}^{\Phi, \varphi_{1}}\left(\mathbb{R}^{n}\right)$ to $\mathcal{M}^{\Psi, \varphi_{2}}\left(\mathbb{R}^{n}\right)$.

3. Assume that $\rho$ is increasing and that $r \mapsto r^{-n} \rho(r)$ is decreasing. Assume also that the conditions (4.2) and (6.6) are satisfied. Let $\varphi_{1}$ and $\varphi_{2}$ be almost decreasing. Then the condition (7.7) is necessary and sufficient for the boundedness of $M_{\rho}$ from $\mathcal{M}^{\Phi, \varphi_{1}}\left(\mathbb{R}^{n}\right)$ to $\mathrm{WM}^{\Psi, \varphi_{2}}\left(\mathbb{R}^{n}\right)$. Moreover, if $\Phi \in \nabla_{2}$, then the condition (7.7) is necessary and sufficient for the boundedness of $M_{\rho}$ from $\mathcal{M}^{\Phi, \varphi_{1}}\left(\mathbb{R}^{n}\right)$ to $\mathcal{M}^{\Psi, \varphi_{2}}\left(\mathbb{R}^{n}\right)$.

Proof. 1. By (6.6), (7.1), (7.6) and the doubling properties of $\varphi_{1}$ and $\Phi^{-1}$ we have

$$
\begin{aligned}
\left\|M_{\rho} f\right\|_{\mathrm{W} \mathcal{M}^{\Psi, \varphi_{2}}} \lesssim & \sup _{x \in \mathbb{R}^{n}, r>0} \varphi_{2}(r)^{-1} \Psi^{-1}\left(r^{-n}\right)\|f\|_{L^{\Phi}(B(x, 2 r))} \\
& +\sup _{x \in \mathbb{R}^{n}, r>0} \varphi_{2}(r)^{-1} \sup _{r<t<\infty}\|f\|_{L^{\Phi}(B(x, 2 t))} \Phi^{-1}\left(t^{-n}\right) \rho(t) \\
\lesssim & \sup _{x \in \mathbb{R}^{n}, r>0} \varphi_{1}(r)^{-1} \Phi^{-1}\left(r^{-n}\right)\|f\|_{L^{\Phi}(B(x, r))} \\
& +\sup _{x \in \mathbb{R}^{n}, r>0} \varphi_{2}(r)^{-1} \sup _{r<t<\infty} \varphi_{1}(t) \rho(t)\|f\|_{\mathcal{M}^{\Phi, \varphi_{1}}} \\
\lesssim & \|f\|_{\mathcal{M}^{\Phi, \varphi_{1}}} .
\end{aligned}
$$

Simply replace $\mathrm{W} L^{\Psi}(B)$ with $L^{\Psi}(B)$ and $W \mathcal{M}^{\Psi, \eta}\left(\mathbb{R}^{n}\right)$ with $\mathcal{M}^{\Psi, \eta}\left(\mathbb{R}^{n}\right)$ for the strong estimate.

2. We will now prove the second part. We utilize (4.5). By Lemma 5.5, we have

$$
\begin{aligned}
\rho(r) & \lesssim|B(0, r)|^{-1}\left\|M_{\rho} \chi_{B(0,2 r)}\right\|_{W L^{1}(B(0, r))} \lesssim \varphi_{2}(r)\left\|M_{\rho} \chi_{B(0,2 r)}\right\|_{\mathrm{WM}^{1, \varphi_{2}}} \\
& \lesssim \varphi_{2}(r)\left\|\chi_{B(0,2 r)}\right\|_{\mathcal{M}^{\Phi, \varphi_{1}}} \lesssim \frac{\varphi_{2}(r)}{\varphi_{1}(r)}
\end{aligned}
$$

3. Since $\varphi_{2}$ is almost decreasing, (77.6) and (7.7) are equaivalent. Then the third statement of the theorem follows from the first and second parts of the theorem. 


\subsection{Adams-type result}

The following theorem gives necessary and sufficient conditions for Adams-type boundedness of the operator $M_{\rho}$ from $\mathcal{M}^{\Phi, \varphi}\left(\mathbb{R}^{n}\right)$ to $\mathcal{M}^{\Psi, \eta}\left(\mathbb{R}^{n}\right)$.

Here we suppose that $\rho$ is an increasing function such that $r \in(0, \infty) \mapsto$ $r^{-n} \rho(r) \in(0, \infty)$ is decreasing.

Theorem 7.3. Let $\Phi$ be a Young function, and let $\varphi \in \mathcal{G}_{\Phi}$ be almost decreasing. Assume that $\rho$ is increasing and that $r \mapsto r^{-n} \rho(r)$ is decreasing. Let $\beta \in(0,1)$, $\eta(t) \equiv \varphi(t)^{\beta}$ and $\Psi(t) \equiv \Phi\left(t^{1 / \beta}\right)$. Then the condition

$$
\rho(t) \lesssim \varphi(t)^{\beta-1}
$$

is necessary and sufficient for the boundedness of $M_{\rho}$ from $\mathcal{M}^{\Phi, \varphi}\left(\mathbb{R}^{n}\right)$ to $\mathrm{W} \mathcal{M}^{\Psi, \eta}\left(\mathbb{R}^{n}\right)$. Moreover, if $\Phi \in \nabla_{2}$, then the condition (7.8) is necessary and sufficient for the boundedness of $M_{\rho}$ from $\mathcal{M}^{\Phi, \varphi}\left(\mathbb{R}^{n}\right)$ to $\mathcal{M}^{\Psi, \eta}\left(\mathbb{R}^{n}\right)$.

As before, we start with an auxiliary pointwise estimate.

Lemma 7.4. Under the assumption of Theorem 7.3 including (17.8), there exists a positive constant $C$ such that, for all $f \in \mathcal{M}^{\Phi, \varphi}\left(\mathbb{R}^{n}\right)$ and all $x \in \mathbb{R}^{n}$,

$$
M_{\rho} f(x) \leq C(M f(x))^{\beta}\|f\|_{\mathcal{M}^{\Phi, \varphi}}^{1-\beta} .
$$

Proof. For arbitrary ball $B=B(x, r)$ we represent $f$ as

$$
f=f_{1}+f_{2}, \quad f_{1}=f \chi_{B}, \quad f_{2}=f-f_{1}, \quad r>0
$$

so that

$$
M_{\rho} f(x) \leq M_{\rho} f_{1}(x)+M_{\rho} f_{2}(x) .
$$

Hence by Lemma 2.7 ,

$$
\begin{aligned}
M_{\rho} f_{2}(x) & =\sup _{t>0} \frac{\rho(t)}{|B(x, t)|} \int_{B(x, t) \backslash(B(x, r))}|f(z)| d z \\
& \leq \sup _{t>r} \frac{\rho(t)}{|B(x, t)|} \int_{B(x, t)}|f(z)| d z \\
& \lesssim \sup _{t>r} \rho(t) \Phi^{-1}\left(|B(x, t)|^{-1}\right)\|f\|_{L^{\Phi}(B(x, t))} \\
& \lesssim\|f\|_{\mathcal{M}^{\Phi, \varphi}} \sup _{t>r} \rho(t) \varphi(t) .
\end{aligned}
$$

Consequently by Lemma 4.1 we have

$$
M_{\rho} f(x) \lesssim \rho(r) M f(x)+\|f\|_{\mathcal{M}^{\Phi, \varphi}} \sup _{t>r} \rho(t) \varphi(t) .
$$


Thus, using the technique in [30, p. 6492] as before and (7.8) we obtain

$$
\begin{aligned}
M_{\rho} f(x) & \lesssim \min \left\{\varphi(r)^{\beta-1} M f(x), \varphi(r)^{\beta}\|f\|_{\mathcal{M}^{\Phi, \varphi}}\right\} \\
& \lesssim \sup _{s>0} \min \left\{s^{\beta-1} M f(x), s^{\beta}\|f\|_{\mathcal{M}^{\Phi, \varphi}}\right\} \\
& =(M f(x))^{\beta}\|f\|_{\mathcal{M}^{\Phi, \varphi}}^{1-\beta},
\end{aligned}
$$

where we have used that the supremum is achieved when the minimum parts are balanced. This shows (7.9).

We prove Theorem 7.3 .

Proof of Theorem 7.3. By using inequality (7.9) and Lemma 6.7 we have, for all balls $B$,

$$
\left\|M_{\rho} f\right\|_{W L^{\Psi}(B)} \lesssim\left\|(M f)^{\beta}\right\|_{W L^{\Psi}(B)}\|f\|_{\mathcal{M}^{\Phi, \varphi}}^{1-\beta}=\|M f\|_{W L^{\Phi}(B)}^{\beta}\|f\|_{\mathcal{M}^{\Phi, \varphi}}^{1-\beta} .
$$

Consequently, by using the boundedness of the maximal operator $M$, we get

$$
\begin{aligned}
& \eta(r)^{-1} \Psi^{-1}\left(|B|^{-1}\right)\left\|M_{\rho} f\right\|_{W L^{\Psi}(B)} \lesssim \eta(r)^{-1} \Psi^{-1}\left(|B|^{-1}\right)\|M f\|_{W L^{\Phi}(B)}^{\beta}\|f\|_{\mathcal{M}^{\Phi, \varphi}}^{1-\beta} \\
& =\left(\varphi(r)^{-1} \Phi^{-1}\left(|B|^{-1}\right)\|M f\|_{W L^{\Phi}(B)}\right)^{\beta}\|f\|_{\mathcal{M}^{\Phi, \varphi}}^{1-\beta} \lesssim\|f\|_{\mathcal{M}^{\Phi, \varphi}} .
\end{aligned}
$$

By taking the supremum over all balls $B$, we get the desired result. Moreover, if $\Phi \in \nabla_{2}$, then we have the strong type estimate.

We will now prove the necessity. We utilize (4.5). By Lemmas 2.6 and 5.5 , we have

$$
\begin{aligned}
\rho(r) & \lesssim \Psi^{-1}\left(r^{-n}\right)\left\|M_{\rho} \chi_{B(0,2 r)}\right\|_{W L^{\Psi}(B(0, r))} \lesssim \eta(r)\left\|M_{\rho} \chi_{B(0,2 r)}\right\|_{\mathrm{WM}^{\Psi, \eta}} \\
& \lesssim \eta(r)\left\|\chi_{B(0,2 r)}\right\|_{\mathcal{M}^{\Phi, \varphi}} \lesssim \frac{\eta(r)}{\varphi(r)} \lesssim \varphi(r)^{\beta-1}
\end{aligned}
$$

Then the proof is complete.

Acknowledgements. The research of F. Deringoz was partially supported by the grant of Ahi Evran University Scientific Research Project (FEF.A4.18.019). The research of V. Guliyev was partially supported by the grant of 1st Azerbaijan-Russia Joint Grant Competition (Agreement number no. EIF-BGM-4-RFTF-1/2017-21/01/1) and by the Ministry of Education and Science of the Russian Federation (the Agreement No. 02.a03.21.0008). Eiichi Nakai was supported by Grant-in-Aid for Scientific Research (B), No. 15H03621, Japan Society for the Promotion of Science. Yoshihiro Sawano was supported by Grant-in-Aid for Scientific Research (C) (16K05209), the Japan Society for the Promotion of Science and by Peoples Friendship University of Russia. 


\section{References}

[1] Adams, D.R.: A note on Riesz potentials, Duke Math. J., 42, 765-778 (1975)

[2] Cianchi, A.: Strong and weak type inequalities for some classical operators in Orlicz spaces. J. London Math. Soc. (2) 60, no. 1, 187-202 (1999)

[3] Deringoz, F., Guliyev, V.S., Samko, S.: Boundedness of maximal and singular operators on generalized Orlicz-Morrey spaces. Operator Theory, Operator Algebras and Applications, Series: Operator Theory: Advances and Applications Vol. 242, 139-158 (2014)

[4] Deringoz, F., Guliyev, V.S., Hasanov, S.G.: A characterization for Adams type boundedness of the fractional maximal operator on generalized Orlicz-Morrey spaces. Integral Transforms Spec. Funct. 28 (4), 284-299 (2017)

[5] Deringoz, F., Guliyev, V.S., Hasanov, S.G.: Characterizations for the Riesz potential and its commutators on generalized Orlicz-Morrey spaces. J. Inequal. Appl. 2016 (248), DOI: 10.1186/s13660-016-1192-z (2016)

[6] Guliyev, V.S., Hasanov, S.G., Sawano, Y., Noi, T.: Non-smooth Atomic Decompositions for Generalized Orlicz-Morrey Spaces of the Third Kind, Acta Appl. Math. 145 (2016), 133-174.

[7] Guliyev, V.S., Ismayilova, A.F., Kucukaslan, A., Serbetci, A.: Generalized Fractional Integral Operators on Generalized Local Morrey Spaces. Journal of Function Spaces, vol. 2015, Article ID 594323, 8 pp

[8] Eridani A., Gunawan H., Nakai E., Sawano Y.: Characterizations for the generalized fractional integral operators on Morrey spaces. Math. Inequal. Appl. 17 (2), 761-777 (2014)

[9] Gala S., Sawano Y., Tanaka H.: A remark on two generalized Orlicz-Morrey spaces, J. Approx. Theory 198, 1-9 (2015)

[10] Hakim D.I., Nakai E., Sawano Y.: Generalized fractional maximal operators and vector-valued inequalities on generalized Orlicz-Morrey spaces, Rev. Mat. Complut. 29, no. 1, 59-90 (2016).

[11] Hästo, Peter A. The maximal operator on generalized Orlicz spaces. J. Funct. Anal. 269, no. 12, 4038-4048 (2015)

[12] Hedberg, L.I: On certain convolution inequalities. Proc. Amer. Math. Soc. 36, 505-510 (1972)

[13] Kawasumi R., Nakai E., Pointwise multipliers on weak Orlicz spaces. https://arxiv.org/abs/1811.02858 
[14] Kokilashvili, V., Krbec M.M.: Weighted Inequalities in Lorentz and Orlicz Spaces, World Scientific, Singapore, 1991.

[15] Maligranda, L.: Orlicz spaces and interpolation, Seminários de Matemática, ISSN 0103-5258; 5 (1989).

[16] Mizuta, Y., Nakai, E., Ohno, T., Shimomura T.: Boundedness of fractional integral operators on Morrey spaces and Sobolev embeddings for generalized Riesz potentials. J. Math. Soc. Japan 62, No. 3, 707-744 (2010)

[17] Nakai, E.: A characterization of pointwise multipliers on the Morrey spaces. Sci. Math. 3 (3), No. 3, 445-454 (2000)

[18] Nakai, E.: On generalized fractional integrals in the Orlicz spaces. Proceedings of the Second ISAAC Congress, Vol. 1 (Fukuoka, 1999), 75-81, Int. Soc. Anal. Appl. Comput., 7, Kluwer Acad. Publ., Dordrecht, 2000.

[19] Nakai, E.: On generalized fractional integrals. Taiwanese J. Math. 5, 587-602 (2001)

[20] Nakai, E.: On generalized fractional integrals in the Orlicz spaces on spaces of homogeneous type. Sci. Math. Jpn. 54, 473-487 (2001)

[21] Nakai, E.: On generalized fractional integrals on the weak Orlicz spaces, $\mathrm{BMO}_{\phi}$, the Morrey spaces and the Campanato spaces. Function spaces, interpolation theory and related topics (Lund, 2000), de Gruyter, Berlin, 2002, 389-401.

[22] Nakai, E.: Generalized fractional integrals on Orlicz-Morrey spaces. In: Banach and Function Spaces. (Kitakyushu, 2003), Yokohama Publishers, Yokohama, 323-333 (2004).

[23] Nakai, E., Sumitomo, H.: On generalized Riesz potentials and spaces of some smooth functions. Sci. Math. Jpn. 54, No. 3, 463-472 (2001)

[24] O’Neil, R.: Fractional integration in Orlicz spaces. I. Trans. Amer. Math. Soc. $115,300-328(1965)$

[25] E. Pustylnik, Generalized potential type operators on rearrangement invariant spaces. Israel Math. Conf. Proc. 13, No 3, 161-171 (1999)

[26] Pérez, C.: Two weighted inequalities for potential and fractional type maximal operators. Indiana Univ. Math. J. 43, 663-683 (1994)

[27] Rao M.M., Ren, Z.D.: Theory of Orlicz Spaces, Dekker 1991. 
[28] Spanne, S.: Some function spaces defined using the mean oscillation over cubes, Ann. Scuola Norm. Sup. Pisa 19 (3), 593-608 (1965)

[29] Sawano, Y., Theory of Besov spaces, Development in Mathematics 56.

[30] Sawano, Y., Sugano, S., Tanaka, H.: Generalized fractional integral operators and fractional maximal operators in the framework of Morrey spaces. Trans. Amer. Math. Soc. 363 (12), 6481-6503 (2011)

[31] Sawano, Y., Sugano, S., Tanaka, H.: Orlicz-Morrey spaces and fractional operators. Potential Anal. 36 (4), 517-556 (2012)

[32] Weiss, G.: A note on Orlicz spaces, Portugal Math. 15, 35-47 (1950)

Fatih Deringoz

Department of Mathematics, Ahi Evran University, Kirsehir, Turkey deringoz@hotmail.com

Vagif S. Guliyev

Department of Mathematics, Dumlupinar University, Kutahya, Turkey

+Institute of Mathematics and Mechanics, Baku, Azerbaijan

+S.M. Nikolskii Institute of Mathematics at RUDN University, Moscow, Russia 117198

vagif@guliyev.com

Eiichi Nakai

Department of Mathematics, Ibaraki University, Mito, Ibaraki 310-8512, Japan

eiichi.nakai.math@vc.ibaraki.ac.jp

Yoshihiro Sawano

Department of Mathematics and Information Sciences, Tokyo Metropolitan University, Minami-Ohsawa 1-1, Hachioji, Tokyo, 192-0397, Japan

+S.M. Nikolskii Institute of Mathematics at RUDN University, Moscow, Russia 117198

yoshihiro-sawano@celery.ocn.ne.jp

Minglei Shi

Department of Mathematics, Ibaraki University, Mito, Ibaraki 310-8512, Japan

18nd2061@vc.ibaraki.ac.jp, stfoursml@gmail.com 